\title{
Universiteit
}

Leiden

The Netherlands

\section{Elastic wave propagation in confined granular systems}

Somfai, E.; Roux, J.N.; Snoeijer, J.H.; Hecke, M.L. van; Saarloos, W. van

\section{Citation}

Somfai, E., Roux, J. N., Snoeijer, J. H., Hecke, M. L. van, \& Saarloos, W. van. (2005). Elastic wave propagation in confined granular systems. Physical Review E, 72(2), 021301. doi:10.1103/PhysRevE.72.021301

Version: $\quad$ Publisher's Version

License: $\quad$ Leiden University Non-exclusive license

Downloaded from: https://hdl.handle.net/1887/66520

Note: To cite this publication please use the final published version (if applicable). 


\title{
Elastic wave propagation in confined granular systems
}

\author{
Ellák Somfai, ${ }^{1, *}$ Jean-Noël Roux, ${ }^{2}$ Jacco H. Snoeijer, ${ }^{1,3}$ Martin van Hecke, ${ }^{4}$ and Wim van Saarloos ${ }^{1}$ \\ ${ }^{1}$ Instituut-Lorentz, Universiteit Leiden, P. O. Box 9506, 2300 RA Leiden, The Netherlands \\ ${ }^{2}$ Laboratoire des Matériaux et des Structures du Génie Civil, Institut Navier, 2 allée Kepler, Cité Descartes, 77420 \\ Champs-sur-Marne, France \\ ${ }^{3}$ ESPCI, 10 Rue Vauquelin, 75231 Paris Cedex 05, France \\ ${ }^{4}$ Kamerlingh Onnes Lab, Universiteit Leiden, P. O. Box 9504, 2300 RA Leiden, The Netherlands \\ (Received 7 August 2004; revised manuscript received 21 April 2005; published 3 August 2005)
}

\begin{abstract}
We present numerical simulations of acoustic wave propagation in confined granular systems consisting of particles interacting with the three-dimensional Hertz-Mindlin force law. The response to a short mechanical excitation on one side of the system is found to be a propagating coherent wave front followed by random oscillations made of multiply scattered waves. We find that the coherent wave front is insensitive to details of the packing: force chains do not play an important role in determining this wave front. The coherent wave propagates linearly in time, and its amplitude and width depend as a power law on distance, while its velocity is roughly compatible with the predictions of macroscopic elasticity. As there is at present no theory for the broadening and decay of the coherent wave, we numerically and analytically study pulse propagation in a one-dimensional chain of identical elastic balls. The results for the broadening and decay exponents of this system differ significantly from those of the random packings. In all our simulations, the speed of the coherent wave front scales with pressure as $p^{1 / 6}$; we compare this result with experimental data on various granular systems where deviations from the $p^{1 / 6}$ behavior are seen. We briefly discuss the eigenmodes of the system and effects of damping are investigated as well.
\end{abstract}

DOI: 10.1103/PhysRevE.72.021301

PACS number(s): 45.70. -n, 43.40.+s, 46.40.Cd, 46.65.+g

\section{INTRODUCTION}

The behavior of granular systems is often strongly influenced by fluctuations and inhomogeneities on the scale of the granular particles, which precludes or complicates the derivation of macroscopic laws from grain-level characteristics. In quasistatic granular packings the strong fluctuations in the intergrain forces are a striking example of this heterogeneity [1]. The fact that the grain-grain contacts which support large forces are usually correlated in a linelike fashion over distances of several particle diameters leads to so called force chains. (In static equilibrium a contact transmitting a large force is often balanced with a single contact on the opposite side of the grain, and this is repeated on several subsequent grains.) These are clearly visible in experiments on twodimensional (2D) packings using photoelastic disks [1] and in simulations, even though their precise definition is not agreed on at present.

More importantly, it is not clear what properties of the granular media are affected by these force chains or indeed by the broad distribution of interparticle forces. It is under debate whether granular media can be described as elastic, and since a static granular packing is a quenched system, an important issue is at what length scale such a continuum (elastic) theory would become appropriate for a granular medium.

An important quantity that can probe these issues is the propagation of sound waves. Surprisingly, even the scaling with pressure of a continuum quantity like the speed of

*Electronic address: ellak@lorentz.leidenuniv.nl sound is still a matter of debate-even though clearly the average interparticle force scales with the pressure, the number of interparticle contacts also increases with pressure, and it is being debated whether this might affect the dependence of the speed of sound on pressure [2-4]. Moreover, one might argue as follows for an interplay between force chain type correlations and sound propagation. In a simple 1D array of grains the group velocity of waves is higher for a more compressed array because of the nonlinearity of the force law [5]. Similarly, a simple calculation shows that in a continuum elastic medium with a hard block embedded in soft surrounding, the small amplitude acoustic waves propagate principally in the hard material. From these observations one might wonder whether the acoustical waves that travel through the granular medium first are transmitted mostly by the strong force chains. If so, one might be able to extract detailed information about the force chains from the transmitted acoustic signal.

In this paper we explore numerically and analytically what ultrasound experiments [6] might tell about the microscopic and mesoscopic features of granular media. Can they tell us anything about the existence of force chains? Can ultrasound probe on what length scale we can view a granular medium as an almost homogeneous random medium? Are there generic features of the propagation of a short pulse which we can uniquely relate to the random nature of a granular pack? These are some of the issues we have in mind in this paper.

Using numerical simulations in $2 \mathrm{D}$ and $3 \mathrm{D}$, we show that sound does not predominantly travel along force chains: the leading part of the wave, which propagates after being excited by a short pulse at one end of the medium, is better characterized as a propagating rough front, like what was 
found in bond-diluted models [7]. Similarly to experiments of this type, we can separate the transmitted acoustic signal into an initial coherent part and a subsequent random part. The coherent wave propagates essentially linearly in time, defining a time-of-flight sound velocity. We have calculated the effective elastic constants for our packings, which under the assumption that the granular packing can be viewed as a continuum directly lead to a simple expression for this velocity. Surprisingly, the observed time-of-flight velocity of the coherent wave is roughly $40 \%$ larger than predicted by continuum elasticity.

We also study the scaling of sound velocity and elastic constants with pressure. For a fixed contact network with Hertzian forces which stay proportional to the pressure $p$, the elastic constants scale as $p^{1 / 3}$ and the sound velocity as $p^{1 / 6}$. In the pressure range studied here we find no discernible deviation from the $p^{1 / 6}$ behavior for the time-of-flight velocity; the situation for the velocities calculated from the effective elastic constants is more convoluted.

Discrete numerical simulations have been used to evaluate the elastic moduli of packings of spherical beads before $[2,8,9]$, from which continuum sound speeds can be deduced. However, to our knowledge, wave propagation has never been directly addressed numerically in a granular material. Measurements of overall elastic properties do not probe the material on the mesoscopic scale and overlook potentially interesting properties such as dispersion and attenuation. Hence the interest and motivation of the present work, which directly copes with the properties of a traveling ultrasonic pulse in a model granular packing.

We find that there are various other interesting aspects of the problem of pulse propagation in granular systems which appear to have received little attention so far: for disordered systems the amplitude of the coherent wave decays as a power law as it propagates, while its width increases linearly. As we are unaware of systematic studies of these issues so far, we also consider, as a reference, the propagation of a sound pulse in 1D chains and in 2D triangular lattices of identical elastic balls. We show that also here both the amplitude and width of the coherent wave behave as power laws of the distance. We calculate both these exponents and the wave front analytically, and show that the broadening of the pulse and the decay of the width are much slower than in the 2D disordered system. A more detailed experimental and theoretical study of these aspects might therefore yield an important way to probe granular media in the future.

This paper is organized as follows. First we review in Sec. II some of the relevant results of experiments and numerical simulations. Then Sec. III describes the details of our numerical model: how the packings are obtained and how the small amplitude oscillations are analyzed. In Sec. IV we present qualitative and quantitative results of our simulations. In Sec. V we compare these results to theoretical models of a 1D chain and a triangular lattice of identical balls. Finally Sec. VI concludes the paper.

\section{ELASTICITY AND SOUND PROPAGATION: EXPERIMENTAL RESULTS}

There have been a number of related experiments that considered sound propagation in granular systems. Because of their direct relevance we briefly review their main results. It is useful to keep in mind that, in principle, two types of experiments can be performed: either one drives the system with constant frequencies and focuses on spectral properties, or one drives the system with short pulses, testing propagative features. Since wave propagation is traditionally described in the framework of macroscopic linear elasticity, we also briefly evoke some of the measurements of elastic moduli as obtained in laboratory experiments.

Liu and Nagel [10-12] studied acoustic sound propagation through an open 3D granular assembly. They prepared a 15-30 grain diameter deep layer of glass beads in an isolated box. In their setup the top surface was free and subject to gravity only. The sound source was a vertical extended plate, embedded within the granular layer, and the detectors were accelerometers of size comparable to the grains. They identified three distinct sound velocities. From the response to a short pulse, the ratio of the source-detector distance and the time of flight gives $c_{\text {tof }}=L / T_{\text {flight }}=280 \pm 30 \mathrm{~m} / \mathrm{s}$, while the dependence of the time of maximum amplitude of the response on source-detector distance yields $c_{\max \text { resp }}$ $=d L / d T_{\max }=110 \pm 15 \mathrm{~m} / \mathrm{s}$. In case of harmonic excitation, the group velocity defined by the frequency dependence of the phase delay is $c_{\text {group }}=2 \pi L d \nu / d \phi=60 \pm 10 \mathrm{~m} / \mathrm{s}$. From these incompatible values they concluded that the granular packing cannot be considered as an effective medium for sound propagation, as the transmission is dominated by the strong spatial fluctuations of force networks. A consequence of this is the extreme sensitivity to small changes (e.g., heating one bead by less than a degree [12]), and the $f^{-2}$ power spectrum in response to harmonic excitation. Many aspects of these experiments have been confirmed by numerical simulations in a model system of square lattice of nonidentical springs [13] and with Hertzian spheres [14].

Jia and co-workers $[15,16]$ measured sound propagation through a confined 3D granular system. They filled a cylinder of radius and length 15-30 grain diameters with glass beads, compacted by horizontal shaking, closed off with a piston, and applied an external pressure on the piston. The sound source was a large flat piezo transducer at the bottom of the cylinder, and the (variable size) detector was at the top wall. They measured the response function for a short pulse, and observed that it can be divided into an initial coherent part, insensitive to the details of the packing, followed by a noisy part, which changed significantly from packing to packing. The ratio of the amplitudes of the two parts of the signal depended on the relative size of the detector and the grains, and also on additional damping (e.g., wet grains [16]).

Gilles and Coste [17] studied sound propagation in a confined 2D lattice of steel or nylon beads. They arranged the beads on a triangular lattice of hexagonal shape, 30 beads on each side, and isotropically compressed the system. This resulted in a regular array of bead centers, but irregular intergrain contacts because of the small polydispersity of the beads. Both the sound source and the sensor were in contact with a single bead at opposite sides of the hexagon. They also found that the response to a short pulse was an initial coherent signal, followed by an incoherent part. While the whole signal was reproducible for a fixed setup, they ob- 
served a high correlation factor between the coherent signals of different packings, and low correlation between the incoherent parts.

Apart from these recent experiments carried out in the physics community, it is worth recalling that many interesting measurements of the elastic or acoustical characteristics of granular materials are to be found in the soil mechanics and geotechnical engineering literature $([18]$ is a recent review stressing the need for sophisticated rheological measurements of soils, including elastic properties). The elastic properties of granular soils have been investigated from quasistatic stress-strain dependencies, as measured with a triaxial [19] or a hollow cylinder [20] apparatus, by "resonant column" devices [21,22] (which measure the frequency of the long wavelength eigenmodes of cylindrically shaped samples), and from sound propagation velocities $[18,20,23-25]$. One remarkable result is the consistency of moduli values obtained with various techniques, provided the applied strain increments are small enough, i.e., typically lower than $10^{-5}$ ). Thus, the agreement between sound propagation and resonant column results is checked, e.g., in [23], while [20] shows consistency between sound propagation velocities and static moduli for very small strain intervals.

In that community, wave velocities are most often deduced from signal time-of-flight measurements between pairs of specifically designed, commercially available piezoelectric transducers known as bender elements $[23,26]$ which couple to transverse modes or bender-extender elements, which also excite longitudinal waves [27]. The typical size of such devices is $\sim 1 \mathrm{~cm}$, sand grain diameters are predominantly in the $100 \mu \mathrm{m}$ range, a typical propagation length (specimen height or diameter) is $10 \mathrm{~cm}$, and confining stresses range from 50 or $100 \mathrm{kPa}$ to several $\mathrm{MPa}$. Those experiments are thus comparable to the ones by Jia and Mills [16], the material being, however, probed on a somewhat larger length scale. The shapes of signals recorded by the receiver are similar (see, e.g., [24,25]).

The soil mechanics literature on wave propagation is chiefly concerned with the measurement of macroscopic elastic moduli, with little interest directed to small scale phenomena. After extracting the wave velocity from the "coherent" part of the signal, using an appropriate procedure, as discussed, e.g., in [28,29], the rest is usually discarded as "scattering" or "near field" effects. Most of these experiments are done in sands, rather than assemblies of spherical balls (see, however, [25]).

To summarize: it appears that granular systems can be considered as an effective medium for the transmission of a short acoustic pulse, if probed on sufficiently large scales and pressures, and if one focuses on the initial part of the response. This wave front is, however, followed by a noisy tail, which is sensitive to packing details, and any quantity or measurement that is dominated by the noisy part, such as $c_{\text {max resp }}$ or $c_{\text {group }}$, will not show the effective medium behavior.

Also, probed on smaller scales or possibly at smaller pressures, the effective medium description appears to become less accurate, even for the initial coherent wave front. The two outstanding questions are thus to identify under what conditions continuum descriptions hold, and if they fail, what other mechanisms come into play.

\section{NUMERICAL MODEL}

Although we performed numerical simulations on 2D and 3D granular packings, this paper mainly contains 2D results. In our setup (most similar to the experiments of Jia et al. $[15,16])$ we have a rectangular box containing confined spheres under pressure. We send acoustic waves through one side of the box and detect force variations at the opposite side.

First we prepare a static configuration of grains. We start with a rectangular box filled with a loose granular gas. For the $2 \mathrm{D}$ simulations the spheres have polydispersity to avoid crystallization: the diameters are uniformly distributed between 0.8 and 1.2 times their average. The bottom of the box is a solid wall, we have periodic boundary conditions on the sides, and the top is a movable piston. We apply a fixed force on the (massive) piston, introduce a Hertz-Mindlin force law, friction included, with some dissipation for the intergrain collisions (see Appendix A), and let the system evolve until all motion stops. Our packings are considered to have converged to mechanical equilibrium when all grains have acceleration less than $10^{-10}$ in our reduced units (defined immediately below). At this point we have a static granular system under external pressure.

In the rest of the paper we use the following conventions. Our unit of length is the average grain diameter. The unit of mass is set by asserting that the material of the grains has unit density. We set the individual grain's modified Young modulus $E^{*}=1$, which becomes the pressure unit (see Appendix A). Since the grains are always 3D spheres, we measure pressure even in the $2 \mathrm{D}$ case as " $3 \mathrm{D}$ pressure," force divided by area, where in 2D the area is length of box side times grain diameter. The speed of sound of the pressure waves inside the grains becomes unity (for zero Poisson ratio). This sets our unit of time, which is about an order of magnitude shorter than the time scale of typical granular vibrations.

Most results are obtained on series of (approximately) square 2D samples containing 600 spheres (their centers being confined to a plane), prepared with friction coefficient $\mu=0.5$ and Poisson ratio $\nu=0$ (see Appendix A). The confining stress $p$ that is controlled in the preparation procedure, and referred to as the pressure throughout this paper, is actually the (principal) stress component $\sigma_{2}$ (or $\sigma_{22}$ in a system of axes for which the coordinate labeled " 2 " varies orthogonally to the top and bottom solid walls). To check for the influence of $p$ on the results, we prepared samples under pressures $p=10^{-7}, 10^{-6}, 10^{-5}$, and $10^{-4}$ (30 samples for each value). To gain statistical accuracy we also prepared an additional series of 1000 samples at $p=10^{-4}$. If the particles are glass beads this corresponds to $7 \mathrm{kPa} \leqslant p \leqslant 7 \mathrm{MPa}$, an interval containing the pressure range within which solid granular packings are usually probed in static or sound propagation experiments. It is worth pointing out that the assembling procedure is repeated for each value of the pressure. It results in a specific anisotropic equilibrium state of the granular assembly, as characterized in Sec. IV A.

To check the robustness of qualitative results on sound propagation, we also studied a few 3D samples, obtained similarly to 2D samples by a compaction of a monodisperse granular gas with a piston compressing in the $z$ direction to 
TABLE I. Static data, corrected for boundary effects: solid fractions, coordination number $z^{*}$, proportion of rattlers $f_{0}$, average normal force, reduced moments [defined in Eq. (5)] of the force distribution, and fabric parameters $F_{2}$ (all contacts) and $F_{2}^{S}$ (strong contacts) defined in Eq. (6). Starred quantities are evaluated on discarding rattlers. Stated error bars correspond, here as in all subsequent tables, to one standard deviation on each side of the mean.

\begin{tabular}{ccccccccccc}
\hline \hline$p$ & $\Phi_{2}$ & $\Phi_{3}$ & $\Phi_{3}^{*}$ & $z^{*}$ & $f_{0}(\%)$ & $\langle N / p\rangle$ & $Z(2)$ & $Z(3)$ & $F_{2}$ & $F_{2}^{S}$ \\
\hline $10^{-7}$ & $0.818 \pm 0.005$ & $0.561 \pm 0.005$ & $0.48 \pm 0.01$ & $3.18 \pm 0.03$ & $15 \pm 2$ & 1.31 & 1.49 & 2.81 & 0.527 & 0.557 \\
$10^{-6}$ & $0.814 \pm 0.004$ & $0.558 \pm 0.005$ & $0.49 \pm 0.01$ & $3.23 \pm 0.04$ & $14 \pm 2$ & 1.27 & 1.50 & 2.83 & 0.524 & 0.560 \\
$10^{-5}$ & $0.809 \pm 0.004$ & $0.554 \pm 0.005$ & $0.50 \pm 0.01$ & $3.31 \pm 0.03$ & $11 \pm 2$ & 1.22 & 1.49 & 2.77 & 0.523 & 0.558 \\
$10^{-4}$ & $0.815 \pm 0.005$ & $0.558 \pm 0.005$ & $0.52 \pm 0.01$ & $3.48 \pm 0.03$ & $7.4 \pm 1.5$ & 1.06 & 1.51 & 2.90 & 0.524 & 0.578 \\
\hline \hline
\end{tabular}

$p=\sigma_{33}=10^{-4}$, and periodic in $x$ and $y$ directions.

In most of the work below, with the exception of Sec. IV F, we study small amplitude oscillations. For this we can use a system linearized around its equilibrium: in the static packing we replace the intergrain contacts with linear springs, with stiffness obtained from the differential stiffnesses (essentially $d F_{t} / d t$ and $d F_{n} / d n$ ) of the individual Hertz-Mindlin contacts (see Appendix A). The equation of motion becomes

$$
M \ddot{u}=-D \mathbf{u},
$$

where for $N$ grains the vector $\mathbf{u}$ contains the $3 N$ coordinates and angles (in 2D) of the particle centers, $\mathrm{M}$ is a diagonal matrix containing grain masses and moments of inertia, and $\mathrm{D}$ is the dynamical matrix containing information about contact stiffnesses and the network topology.

Then we solve the eigenproblem of the linear spring system and write the oscillation of the grains as the superposition

$$
\mathbf{u}(t)=\sum_{n} a_{n} \hat{\mathbf{u}}^{(n)} \sin \left(\omega_{n} t\right) .
$$

The amplitudes $a_{n}$ are obtained by the projection of the initial condition onto the eigenmodes. When the force on the top wall is calculated, we calculate the coupling $b_{n}$ of the given mode with the wall, so the force is

$$
F_{\text {top }}=\sum_{n} a_{n} b_{n} \sin \left(\omega_{n} t\right) .
$$

Typically we will look at the transmission of a short pulse through the granular packing. We send in a $\delta$ pulse, corresponding to wideband excitation. This corresponds to the following initial condition: at $t=0$ the grains in contact with the bottom wall have a velocity proportional to the stiffness of the contact with the wall. This is equivalent to an infinitesimally short square pulse: raising the bottom wall for an infinitesimally short time and lowering it back. The amplitude of the pulse does not matter as all the calculations are linear (except in Sec. IV F). The quantities we wish to study are the resulting force variations on the top wall (the "signal"), defined as the force exerted by the vibrating grains on the top wall minus the static equilibrium force:

$$
F_{\text {sig }}(t)=F_{\text {top }}(t)-F_{\text {top }}\left(0^{-}\right) .
$$

\section{NUMERICAL RESULTS}

In this section we will present the results of extensive numerical simulations of sound propagation in granular media. We first characterize the geometric state of the packing and measure its static properties, including the tensor of elastic moduli. Then we report wave propagation simulations, showing that in our system, as in the experiments of Jia et al. $[15,16], F_{\text {sig }}$ is composed of a coherent initial peak and an incoherent disordered tail. We find that force lines are fairly irrelevant for the pulse propagation, and we will show that the decay and spreading of the initial peak follow packingdetail-independent scaling laws. Furthermore we study the pressure dependence of the transmission velocity of the initial pulse, which is compared to the one deduced from static elastic properties. We close this section by a short discussion of the spectrum and eigenmodes that determine the sound propagation, and briefly study the effect of dissipation.

\section{A. Statics}

We first characterize the system by its geometric and static properties, a necessary step as sound propagation is sensitive to the internal state of a granular packing. Our preparation procedure yields values given in Table I for solid fractions $\Phi_{2}$ (2D definition) and $\Phi_{3}$ (3D definition, in a one diameter thick layer) and proportion of rattlers (particles that transmit no force) $f_{0}$. For sound propagation, the relevant mass density, with our choice of units, is equal to the $3 \mathrm{D}$ solid fraction $\Phi_{3}^{*}$ of nonrattler grains. Values for those parameters are given on accounting for boundary effects: measurements exclude top and bottom layers of thickness $l$, and we checked that results became $l$ independent for $l \geqslant 3$. Table I also gives the bulk values (corrected for wall effects) of the mechanical coordination number $z^{*}$ (i.e., the average number of force-carrying contacts per nonrattler particle), the average normal contact force $N$, normalized by the pressure, and some reduced moments of the distribution of normal contact forces, defined as

$$
Z(\alpha)=\frac{\left\langle N^{\alpha}\right\rangle}{\langle N\rangle^{\alpha}} .
$$

$Z$ values are characteristic of the shape of the force distribution [the wider the distribution, the farther from 1 is $Z(\alpha)$ for any $\alpha \neq 1]$.

The renewal of compression procedure from a granular gas at each value of $p$ is responsible for the apparent absence 
TABLE II. Stress ratio and elastic moduli, as defined in Eq. (7), for the four investigated pressures with $\mu=0.5$ and $\nu=0$.

\begin{tabular}{cccccc}
\hline \hline Pressure & $\sigma_{1} / \sigma_{2}$ & $C_{11}$ & $C_{12}$ & $C_{22}$ & $C_{33}$ \\
\hline $10^{-7}$ & $0.79 \pm 0.06$ & $(9.9 \pm 0.9) \times 10^{-4}$ & $(8.1 \pm 0.4) \times 10^{-4}$ & $(13.9 \pm 0.8) \times 10^{-4}$ & $(1.2 \pm 0.4) \times 10^{-4}$ \\
$10^{-6}$ & $0.79 \pm 0.06$ & $(2.3 \pm 0.2) \times 10^{-3}$ & $(1.7 \pm 0.08) \times 10^{-3}$ & $(3.2 \pm 0.2) \times 10^{-3}$ & $(0.35 \pm 0.07) \times 10^{-3}$ \\
$10^{-5}$ & $0.78 \pm 0.06$ & $(5.4 \pm 0.5) \times 10^{-3}$ & $(3.5 \pm 0.2) \times 10^{-3}$ & $(7.4 \pm 0.3) \times 10^{-3}$ & $(1.1 \pm 0.2) \times 10^{-3}$ \\
$10^{-4}$ & $0.71 \pm 0.04$ & $(1.35 \pm 0.09) \times 10^{-2}$ & $(6.8 \pm 0.4) \times 10^{-3}$ & $(1.88 \pm 0.08) \times 10^{-2}$ & $(4.0 \pm 0.5) \times 10^{-3}$ \\
\hline \hline
\end{tabular}

of systematic density increases over the range of pressure studied here.

As observed in other studies (see, e.g., [9,30]), a direct compression of a granular gas with friction yields rather loose samples, with a low coordination number. The minimum value of $z^{*}$ deduced from the condition that the number of unknown contact forces should be at least equal to the number of equilibrium equations for active particles is three in $2 \mathrm{D}$, and the values given in Table I are only barely larger for the smaller values of $p$. This means that our samples have a low degree of force indeterminacy at $p=10^{-7}$. Complete absence of force indeterminacy is obtained with frictionless beads in the limit of zero pressure [31-34]. With frictional contacts, it appears that some force indeterminacy persists even in the limit of zero pressure $[30,33,35]$.

The fraction of rattlers is very large (up to $15 \%$ ), when $z^{*}$ is close to 3 , while the number of rattlers fastly decrease as $z^{*}$ increases with $p$. We also computed the state of stress in the samples: due to the assembling procedure, the principal directions are horizontal (coordinate $x$, principal value $\sigma_{1}$ ) and vertical (coordinate $y$, principal value $\sigma_{2}$ ), with a larger vertical stress, $\sigma_{1} / \sigma_{2}<1$. This ratio (Table II) stays constant, within statistical error bars, as the controlled confining stress $\sigma_{2}=p$ is varied, except at the highest pressure $p=10^{-4}$. (Stresses are readily evaluated with the usual formula

$$
\sigma_{\alpha \beta}=\frac{1}{V} \sum_{i<j} F_{i j}^{\alpha} r_{i j}^{\beta},
$$

where $F_{i j}^{\alpha}$ is the $\alpha$ component of the force exerted on particle $i$ by particle $j$, and the vector $\mathbf{r}_{i j}$, pointing from the center of particle $i$ to the center of $j$, should account for periodic boundary conditions and involve "nearest image" neighbors.)

The force network of a typical granular configuration of 600 grains prepared at pressure $p=10^{-4}$ and friction coefficient $\mu=0.5$ is shown in Fig. 1(a). We show the stiffness network on Fig. 1(b) and the histogram of forces and stiffnesses on Fig. 1(c), to which we will come back shortly. It is interesting to note that the shape of the force distribution changes very little in the investigated pressure range: values of reduced moments $Z$, listed in Table I, hardly change with pressure. In Ref. [30], a "participation number" $\Pi$ was defined, as an indicator of the width of the force distribution, or the "degree of localization" of stresses on force chains. In fact, one would have $\Pi=1 / Z(2)$ if we had defined $Z$ with the magnitude of the total contact force instead of its sole normal component. Makse et al. [30] found that $\Pi$ increases linearly with $\ln p$, until it saturates at $p \geqslant 10^{-5}$. Our contradictory observation of a nearly constant $Z(2)$ is likely due to our compressing the system anew from a granular gas at each $p$, instead of quasistatically increasing $p$ in a previously assembled solid sample.

The anisotropy of the contact network (which carries anisotropic stresses) is apparent in the histogram of contact angles and the force histograms. Figure 2 shows the histogram of contact directions. We also plot the contribution from strong (contact force larger than the average) and weak (force smaller than average) contacts. Due to the assembly procedure there is an anistropic stress field, resulting in a bias of the strong contacts toward the vertical direction. Let us recall that this is also the principal direction corresponding to the largest eigenvalue of the stress tensor. As a direct consequence of the force anisotropy, the small forces are biased in the opposite direction, although this effect is less pronounced [36]. One way to quantitatively assess the importance of this anisotropy is to compute the fabric parameter

$$
F_{2}=\left\langle n_{y}^{2}\right\rangle,
$$

where $n_{y}$ is the vertical coordinate of the unit normal vector and the average runs over the contacts. The departure of $F_{2}$ from its isotropic value $1 / 2$ measures the anisotropy. Table I gives $F_{2}$ for the different pressure levels, along with parameter $F_{2}^{S}$, obtained on counting only the contacts that carry larger than average forces. Once again, the level of anisotropy does not depend on pressure, except for a slight difference at $p=10^{-4}$, in which case it is a little larger (consistently with the larger stress anisotropy).

If, on long length scales, the material can be considered as an ordinary homogeneous 2D material with two orthogonal symmetry axes, the granular packing has four independent macroscopic elastic moduli, which relate the three independent coordinates of the symmetric stress tensor $\sigma_{i j}$ to the three coordinates of the symmetric strain tensor $\epsilon_{i j}$ as

$$
\left[\begin{array}{l}
\sigma_{11} \\
\sigma_{22} \\
\sigma_{12}
\end{array}\right]=\left[\begin{array}{ccc}
C_{11} & C_{12} & 0 \\
C_{12} & C_{22} & 0 \\
0 & 0 & 2 C_{33}
\end{array}\right]\left[\begin{array}{l}
\epsilon_{11} \\
\epsilon_{22} \\
\epsilon_{12}
\end{array}\right] .
$$

In Eq. (7), indices 1 and 2 correspond to the horizontal (periodic) and vertical (along which the normal stress is controlled) directions on the figures. Counting positively shrinking deformations and compressive stresses, the strain components should be related to the displacement field $\mathbf{u}$ as 

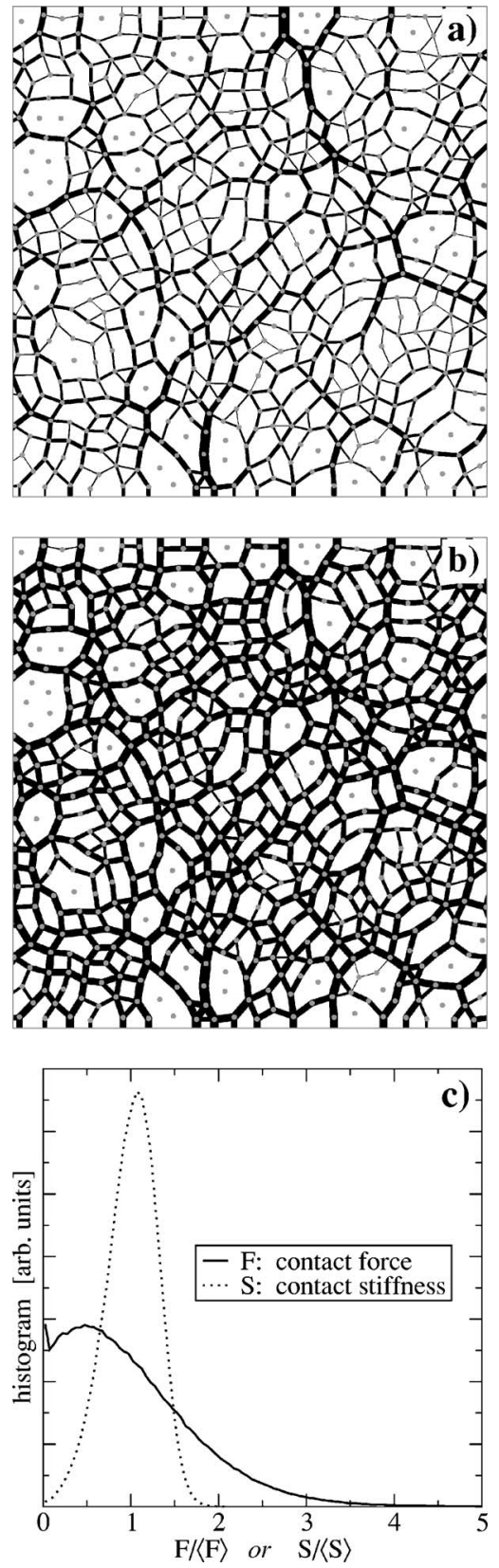

FIG. 1. (a) Snapshot of a force network at pressure $p=10^{-4}$. Linewidths are proportional to the force between the grains, grain centers are plotted with gray dots. Only the normal forces are plotted, the tangential (frictional) are not. (b) The stiffness network of the same configuration. Linewidths are proportional to the stiffness $d F_{n} / d n$ of the contact (normal part). While the force network shows considerable spatial fluctuations, the stiffness network is much more homogeneous. (c) Histogram of the normal contact forces and contact stiffnesses of 1000 configurations. The areas under the two curves are the same. The plot shows that the stiffness is more narrowly distributed, a feature discussed in more detail in Sec. IV B.

$$
\epsilon_{i j}=-\frac{1}{2}\left(\frac{\partial u_{i}}{\partial x_{j}}+\frac{\partial u_{j}}{\partial x_{i}}\right) .
$$

The principal stress ratios $\sigma_{1} / \sigma_{2}$ and apparent values of the four moduli introduced in Eq. (7), obtained on imposing glo-

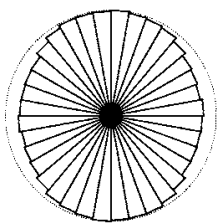

all contacts

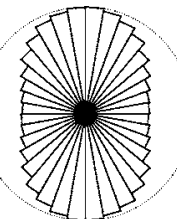

strong contucts

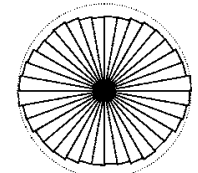

weak contacts
FIG. 2. Histogram of contact directions at pressure $p=10^{-4}$. The diagrams show the average of 1000 independent configurations, 600 grains each, and only bulk contacts are counted (at least three particle diameters away from walls). The strong contacts (contact force larger than the average) show significant anisotropy: vertical directions are favored. The weak forces are much more isotropic, although slightly more of them are horizontal than vertical (as described in [36]). Recall that the piston compressed the grains in the vertical direction.

bal strains on the rectangular cell containing the samples, are given in Table II. To check for possible length scale effects on static elasticity (in view of the small system size), samples were submitted to inhomogeneous force fields, or to various local conditions on displacements. The result of these computer experiments, described in Appendix B, is that constants $C_{22}$ and $C_{33}$ are already quite well defined on the (modest) scale of the 600-sphere samples (typically $24 \times 25$ ). The assumption that nonuniform stress and strain fields, which vary on the scale of a fraction of the sample size, are related by (7), with the elastic constants of Table II, predicts results which approximately agree with numerical tests on our discrete packings, in spite of their moderate size. This agreement is better for the longitudinal constant $C_{22}$ than for the shear modulus $C_{33}$, and improves on increasing $p$.

The conclusion that macroscopic elasticity applies even at moderate length scales is in agreement with the results by Goldhirsch and Goldenberg on homogeneously forced disordered packings [37]. However, when probing the response to localized forces (which perturb the system inhomogenously even in the elastic limit) those authors identified a larger length scale of about 100 diameters in order to recover macroscopic elasticity [38]; these differences might also be due to the fact that their study concerned frictionless quasiordered systems. We should keep in mind also that Goldhirsch and Goldenberg looked at the full spatial dependence of the elastic response, while we extracted only global elastic quantities. One may also note (see, e.g., [39]) that constitutive laws are obtained with numerical simulations of disordered granular samples in the quasistatic regime with relatively small finite-size effects when the number of particles is above 1000 , and that the level of uncertainty and fluctuations is further reduced on investigating the response to small perturbations on a fixed contact network. Tanguy et al. [40] studied the finite-size effects on the elastic properties of $2 \mathrm{D}$ Lennard-Jones systems at zero temperature. While large sample sizes $(N \sim 10000$ particles $)$ were necessary for the low-frequency eigenmodes to resemble the macroscopic predictions (an issue we shall return to in a subsequent paper) they did observe apparent elastic constants, as measured on globally deforming the sample, to converge quickly (for $N$ 100) to their macroscopic limit. The observation of mac- 


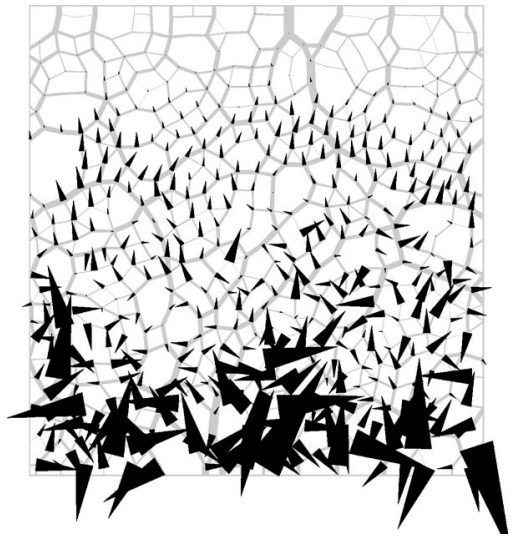

FIG. 3. Snapshot of the oscillations. The lengths of the arrows show the (magnified) displacement of the grains from their equilibrium position. One can see the localized large amplitude oscillations of the grains near the bottom wall (source), and a smaller amplitude homogeneous wave traveling toward the top wall (detector). At the time of the snapshot, $t=80$, the wave almost reached the top wall.

roscopic elastic behavior (with some limited accuracy) in an assembly of 600 particles is not really surprising in this context.

Assuming macroscopic elasticity to hold in our samples, elastic constants $C_{22}$ and $C_{33}$ determine velocities of longitudinal $\left(c_{\ell}\right)$ and transverse $\left(c_{t}\right)$ waves propagating in direction 2 (normal to top and bottom walls), as

$$
c_{\ell}=\sqrt{\frac{C_{22}}{\Phi_{3}^{*}}} \text { and } c_{t}=\sqrt{\frac{C_{33}}{\Phi_{3}^{*}}} .
$$

It should be pointed that the different elastic constants do not exhibit the same scaling with the pressure. Most notably, the ratio of shear modulus $C_{33}$ to longitudinal modulus $C_{22}$ [or the ratio of the corresponding wave velocities, according to (8)] steadily increases with $p$. We shall return to this issue, and compare different predictions for sound velocities and their pressure dependence, in Sec. IV D and in the discussion.

\section{B. Wave propagation: Qualitative observations}

We now turn our attention to the pulse propagation. Before studying $F_{\text {sig }}$, we will discuss here an example of the spatial structure of the propagating pulse. Our first observation is that acoustical waves do not correlate in any obvious way with the existence of force-chain-like configurations. A snapshot of the grain oscillations shortly after the system is "kicked" is shown inFig. 3.

This figure clearly shows that the naive idea that the acoustic waves would follow the strongest granular force chains is false. Instead, one can see the propagation of a rough wave front. One reason we can immediately point to is that even though the forces of the intergrain contacts exhibit a strong spatial fluctuation, the stiffness is much more homogeneous [see Fig. 1(b)]. This can be understood simply as follows. Consider a force law which in scaled units reads $F_{n}=n^{\beta}$, where $n$ is the normal deformation. The stiffness $s$ is then simply given by $d F_{n} / d n=\beta n^{\beta-1}$. Clearly, for $\beta=1$ (corresponding to the $2 \mathrm{D}$ Hertzian force law), all the stiffness values are the same. For the Hertz-Mindlin law, $\beta=3 / 2$, and we find that the stiffnesses are proportional to the cubic root of the contact forces, leading to the rather homogeneous stiffness network shown in Fig. 1(b). So if we compare two links with forces differing by a factor 8 , the corresponding stiffnesses only differ by a factor 2 , and the sound speedsproportional to the square root of the stiffness-differ only by a factor of $\sqrt{2}$. Even though the contact forces follow a wide distribution, the stiffness distribution is strongly peaked [see Fig. 1(c)]. Although this is a rather trivial observation for Hertzian contacts, we are not aware of its being explicitly mentioned in the literature.

An additional reason for the weak effect of force chains on the sound propagation may be that the disorder of the grains is significant: on a force chain with weak side links the oscillation quickly spreads into its neighborhood, resulting in a more homogeneous base of the oscillations. Anyway, the conclusion we can draw here is that the force chains are not relevant for the evolution of the initial wave front.

\section{The coherent wave front}

Let us now study the experimentally accessible signal $F_{\text {sig. }}$. The time dependence of this signal is shown in Fig. 4(a). Clearly $F_{\text {sig }}$ can be thought to be composed of an initial peak followed by a long incoherent tail. One can see that for configurations that are similar in overall geometry but statistically independent, the initial first cycle of the signal is very similar, but the following part is strongly configuration dependent. The time dependence of the signal is very reminiscent of the traces measured by Jia et al. [15] in their ultrasound experiments. Following the nomenclature introduced by these authors, we call the first part of the signal the coherent part. In the ensemble average only the coherent part of the signal shows up (plus its later weaker echoes) [see Fig. 4(b)]. The random part of the signal contributes to the rootmean-square deviation. We also found that qualitatively, $F_{\text {sig }}$ is very similar for $2 \mathrm{D}$ frictional, $2 \mathrm{D}$ frictionless and $3 \mathrm{D}$ frictionless systems [see Figs. 4(a)-4(d)].

We will now focus on the initial peak of $F_{\text {sig }}$, and determine for this coherent wave front its propagation velocity, and the time evolution of its shape. We have only measured the time dependence of the signal at a fixed distance, and a qualitative picture of the evolution of the coherent wave can be extracted from a sequence of measurements at varying source-detector distance. This is shown in Fig. 5: during the propagation of the signal (as it arrives later at longer distances), the coherent part's amplitude decreases, and its width increases.

Now we look at $F_{\text {sig }}$ quantitatively. As shown in the inset of Fig. 6, we characterize the coherent peak by three points: its peak location, its first $10 \%$ of peak value, and its first zero crossing. In Fig. 6 we show, for various source-detector distances, the times at which these three characteristics can be observed at the detector. In reasonable approximation, the time of flight depends linearly on the source-detector distance, although the upward curving of the data suggests that 

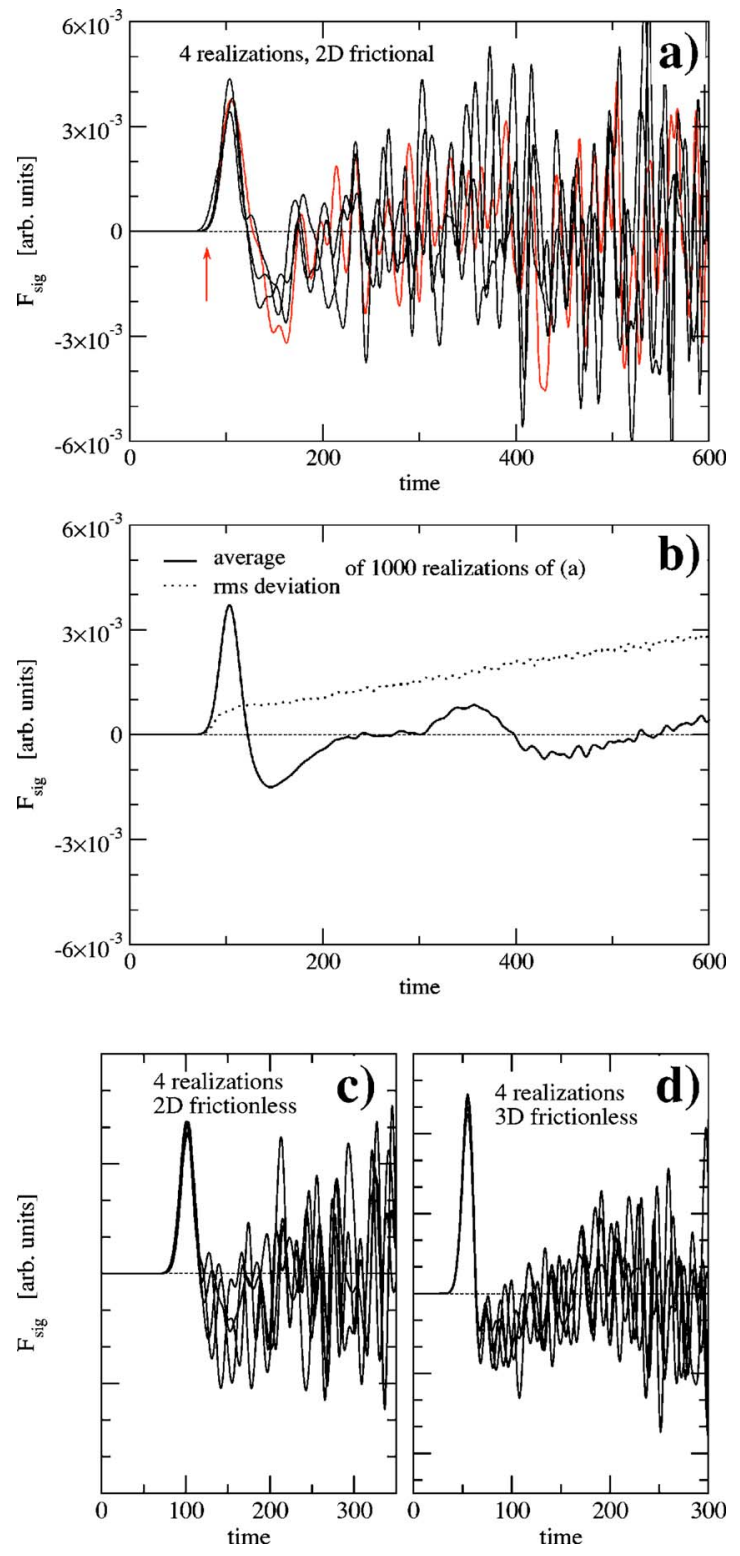

FIG. 4. (Color online) The signal $F_{\text {sig }}$, the extra force exerted by moving grains on the top wall. (a) Four independent 2D frictional configurations are shown; the signal corresponding to the packing of Fig. 1 is plotted in red. The arrow indicates the time of the snapshot of Fig. 3. (b) The ensemble average and root-mean-square deviation of 1000 independent configurations. The first cycle of the oscillations is almost the same on all configurations (we call this the coherent part of the signal), while the following part is very much configuration dependent. The ensemble average only contains the coherent part plus some weak broadened sign of multiple reflections on the top and bottom walls. (c) 2D frictionless and (d) 3D frictionless systems exhibit similar behavior. Time (as well as length in subsequent figures) is denoted in dimensionless units; see Sec. III for details.

for small systems the propagation velocity appears larger than for large systems. We define the time-of-flight velocity $c_{\text {TOF }}$ by measuring the difference of the arrival times (at $10 \%$ of the peak's level) for source-detector distances of 8 and 25. The velocity thus defined can be measured in reasonable

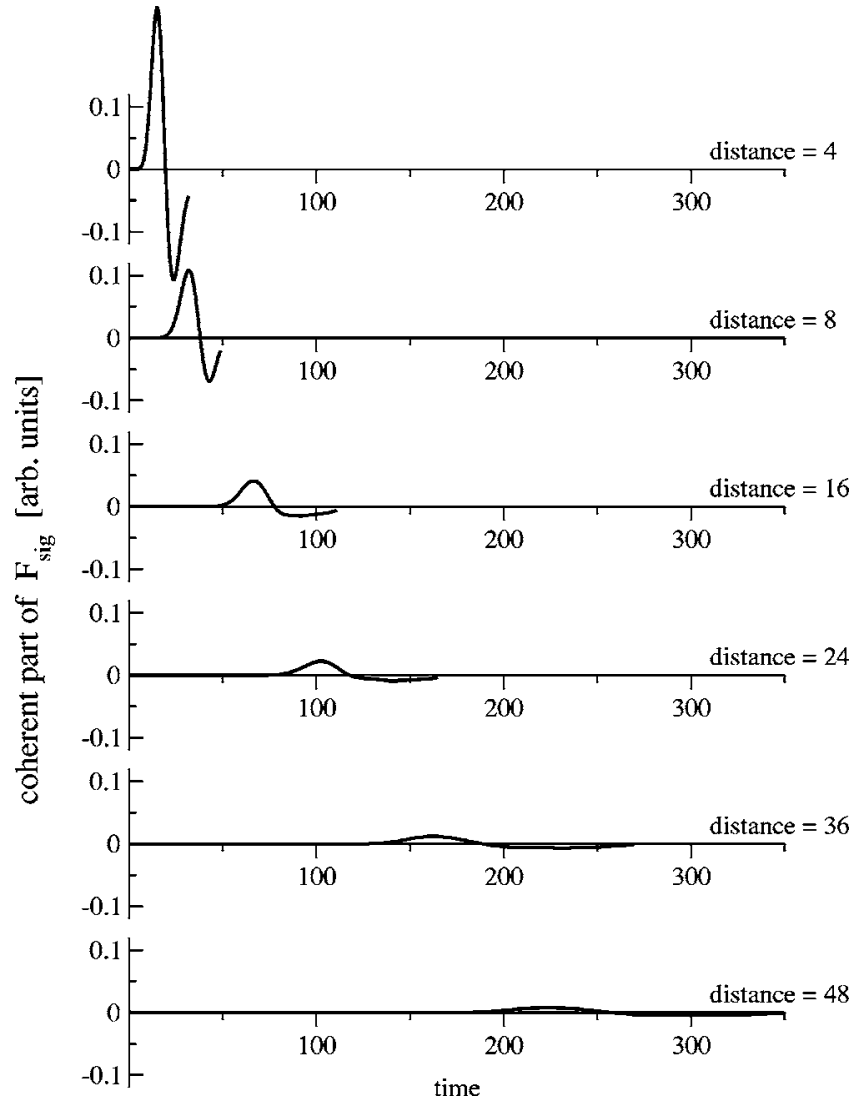

FIG. 5. The coherent part of the signal in containers of varying height (source-detector distance). For taller containers the signal arrives later with decreased amplitude and increased width. These are quantitatively analyzed in the next few figures.

small systems (containing 200 and 600 particles respectively), while on the other hand being quite close (within $10 \%)$ to the large scale velocity. Based on this definition of time of flight, we have a sound speed $c_{\mathrm{TOF}}=0.26$ in our units, at pressure $p=10^{-4}$.

In Fig. 7 we plot the scaling of the amplitude and the width of the coherent part of the signal. The amplitude is well approximated with a power law $A \sim L^{-\gamma}$; for the $2 \mathrm{D}$ simulations $\gamma \approx 1.5$. The width of the coherent part of the signal increases with distance also as a power law, $\sim L^{\alpha}$. For the $2 \mathrm{D}$ simulations the increase is close to linear, $\alpha \approx 1$.

We are not aware of any prediction or previous analysis of these exponents $\gamma$ and $\alpha$ for polydisperse random packings. In order to put these results into perspective, it is important to keep in mind that $F_{\text {sig }}$ is not the amplitude of the wave motion in the medium, but the resulting force on the boundary at the other edge. Since the force is proportional to the local stretching, i.e., the derivative of the amplitude of the wave, $\gamma$ is not the exponent with which the wave amplitude itself decays [see also the discussion around Eq. (15)].

We have compared this behavior with the behavior of propagating pulses in a one-dimensional chain of balls. Even in this simple system, dispersion effects (wave number dependence of the frequency of the waves) give rise to nontrivial exponents - as we shall discuss in more detail in Sec. $\mathrm{V}$, both the exponent and the shape of the pulse can be de- 


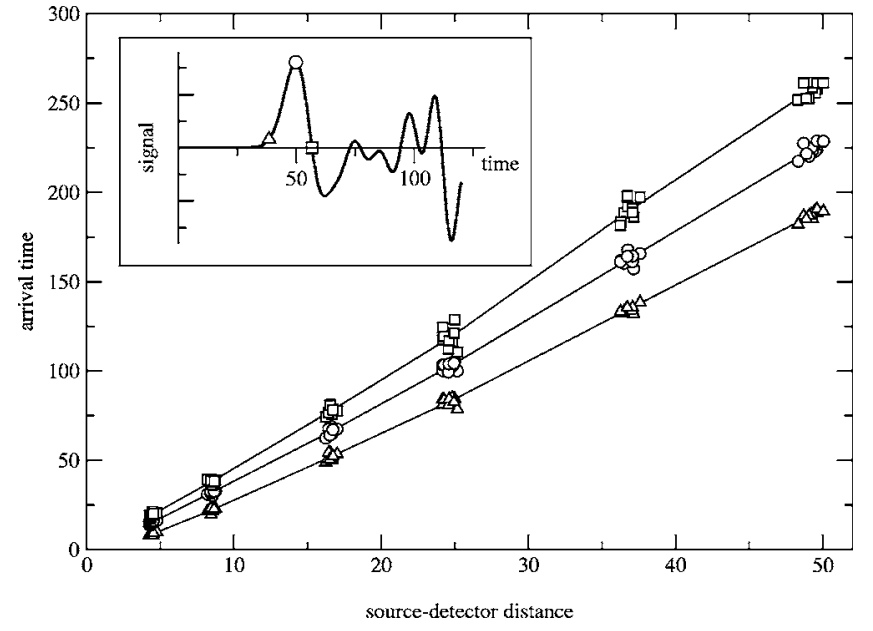

FIG. 6. The arrival time of the coherent part of the signal as a function of the source-detector distance. The inset shows the definition of the symbols: leading edge at $10 \%$ of the first peak height $(\triangle)$, the first peak $(\bigcirc)$, and the first zero crossing of the signal $(\square)$. All three characteristic points of the signal have a linear timedistance relation. The slope of the time-distance plot of the leading edge defines a time-of-flight velocity $c_{\mathrm{TOF}}=0.25$.

termined analytically. We collect the exponents $\gamma$ and $\alpha$ in Table III. An important lesson from the 1D analysis is that the decay exponent $\gamma$ is not universal, as it depends on the precise shape of the initial pulse: $\gamma=2 / 3$ for our usual initial condition (equilibrium position but nonzero velocity next to the wall at $t=0)$, and $\gamma=1$ if the initial condition is zero velocity but nonzero displacement at the wall (not plotted). If we allow polydispersity in the $1 \mathrm{D}$ chain, the scaling appears to have a larger exponent depending on the magnitude of the polydispersity, although from the data shown in Fig. 7 we cannot draw a definite conclusion.

In conclusion, the main qualitative differences between the $1 \mathrm{D}$ results and those for the coherent pulse in the disordered $2 \mathrm{D}$ packings is that (i) in the $1 \mathrm{D}$ chain the first pulse broadens as $t^{1 / 3}$ whereas the pulse in the disordered 2D medium broadens linearly; (ii) the amplitude of the pulse decays much faster in the disordered medium than in the 1D chains (in other words, $\gamma$ is larger).

\section{Speed of sound, elastic moduli, and pressure dependence}

In this section we turn our attention to the sound speed, and in particular study its variation as a function of the confining pressure $p$. The main quantity is the time-of-flight velocity obtained from the propagation of the coherent pulse $c_{\mathrm{TOF}}$ (see Sec. IV C). It should be compared to the values of transversal $\left(c_{t}\right)$ and longitudinal $\left(c_{\ell}\right)$ wave speeds that are deduced [Eq. (8)] from the apparent elastic moduli of Table II. We also compare our results to experimental data for sound propagation; since some experiments have been performed on regular packings, we also have studied these analytically and numerically (see Sec. V). An overview of these various propagation velocities as function of pressure is shown in Fig. 8. Let us first discuss the scaling of $c_{\mathrm{TOF}}, c_{\ell}$ and $c_{t}$ [as defined in (8)] with $p$. Recall that for a fixed
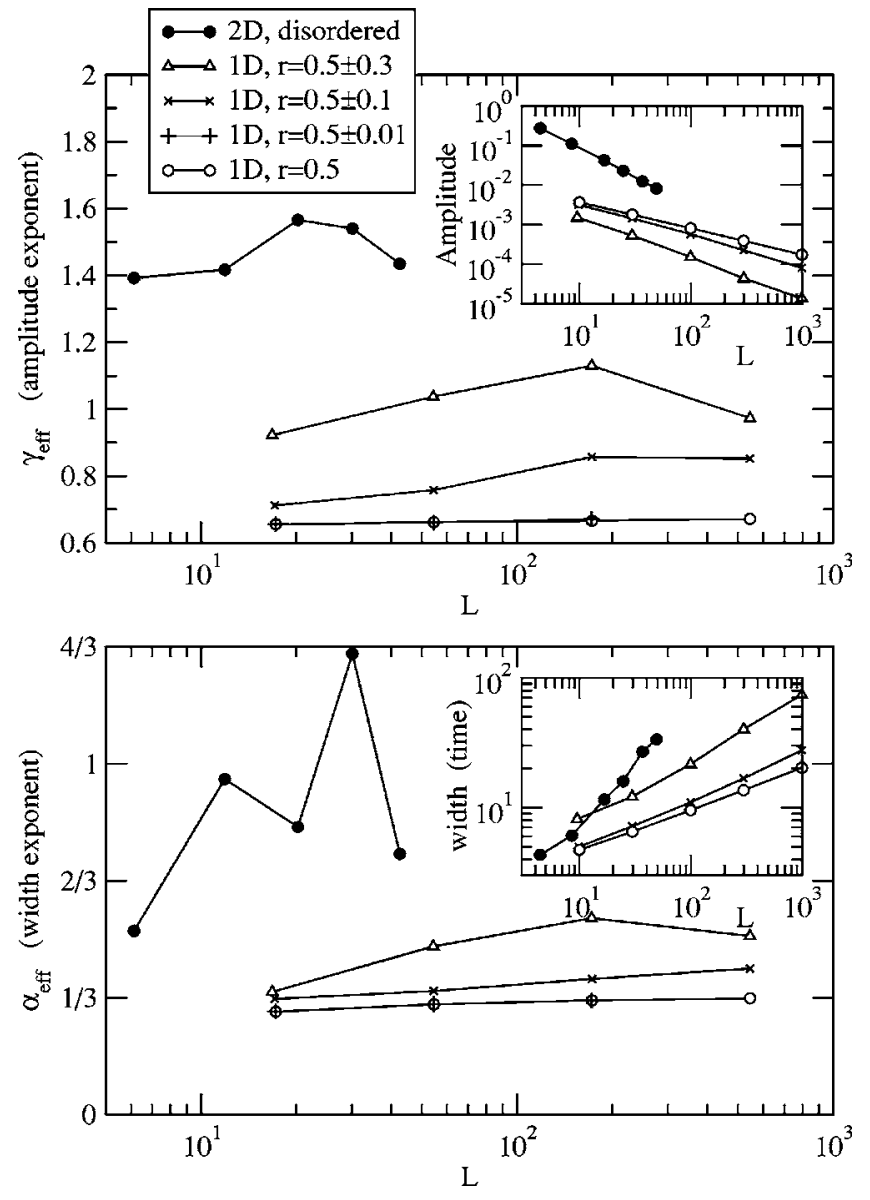

FIG. 7. The scaling of the amplitude and width of the coherent part of the signal with the source-detector distance $L$. Upper panel: the amplitude follows roughly $A \sim L^{-\gamma}$ (inset). In the main panel we plot the effective value of the exponent: $\gamma_{\text {eff }}=d \log _{10} A / d \log _{10} L$. The symbols are 2D disordered (full circles), 1D chain of identical balls (open circles), and 1D chain of polydisperse balls (other symbols, with varying polydispersity). Lower panel: the width of the coherent part of the signal increases with distance: width $\sim L^{\alpha}$ (inset). In the main panel here also the effective exponent $\alpha_{\text {eff }}$ $=d \log _{10} W / d \log _{10} L$ is plotted.

contact network with Hertzian forces that stay proportional to the pressure $p$, the sound velocity scales as $p^{1 / 6}$. We find here that $c_{\mathrm{TOF}}$ follows this scaling quite accurately, while $c_{\ell}$ appears to be growing slightly faster as $p^{0.18}$. Surprisingly, data for the velocity $c_{t}$ of transverse waves abide by a different scaling, $c_{t} \sim p^{0.23}$; we do not know the reason for this behavior.

Since the coherent wave is essentially longitudinal in nature, one should compare $c_{\ell}$ and $c_{\text {TOF }}$. Even though both quantities scale rather similarly, $c_{\mathrm{TOF}}$ is roughly $40 \%$ larger than $c_{\ell}$. As discussed in Sec. IV C, our definition of $c_{\mathrm{TOF}}$ is based on measurements in relatively small systems, and from a few simulations in larger systems we found that this may overestimate $c_{\mathrm{TOF}}$ by some $10 \%$. In addition, if we do not measure the first arrival of the signal, but instead measure the first peak location, or the first zero crossing, $c_{\mathrm{TOF}}$ will go down substantially.

Furthermore, it seems that the pulse propagation with our method of excitation does not probe the material on the long- 


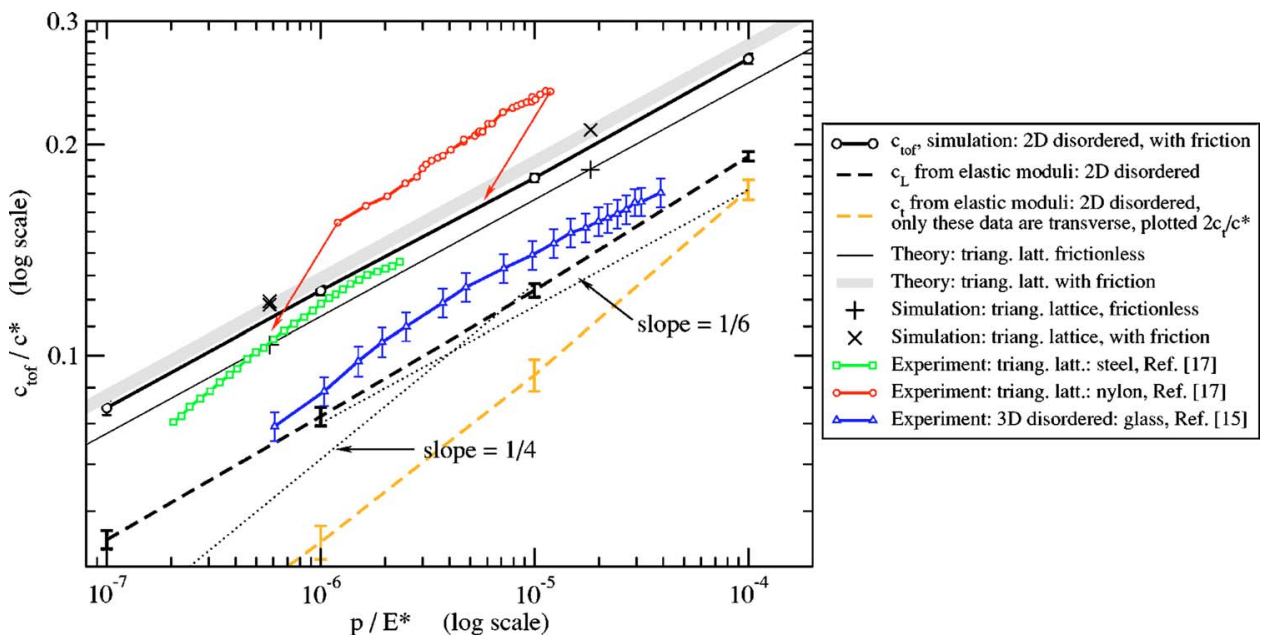

FIG. 8. (Color online) The pressure dependence of the various sound speeds. The main data are the results for $c_{\mathrm{TOF}}$ obtained from our simulations, which show perfect $p^{1 / 6}$ scaling. Velocities $c_{\ell}$ and $c_{t}$ deduced from elastic moduli, as in Eq. (8), are smaller; one would have expected that $c_{\mathrm{TOF}} \sim c_{\ell} . c_{t}$ is much smaller and has been multiplied by 2 to fit within the scale of the plot. The theoretical curves for triangular lattice are Eqs. (18) (frictionless) and (19) (frictional). For the latter there is a slight variation depending on the Poisson ratio of the grain's material: the gray band corresponds to the range $0 \leqslant \nu \leqslant 0.5$. Simulations for the frictionless triangular lattice (+) show excellent agreement with Eq. (18). For the frictional case $(\times)$ the simulation shows significant finite-size scaling: it should approach the top side of the gray band (we used $\nu=0$ and size $L=24$, but for $p=5 \times 10^{-7}$ a larger system $L=160$ is also plotted). The simulation for 2D disordered frictional case $(\bigcirc)$ shows results very similar to the triangular lattice. For comparison we also show three experimental data sets [recall the pressure and velocity scales: $E^{*}=E /\left(1-\nu^{2}\right)$ and $c^{*}=\sqrt{E^{*} / \rho}$ ]: triangular lattice of steel spheres [(green) $\square$, from [17]], triangular lattice of nylon balls [(red) $\bigcirc$, also from [17]; see text for explanation of the arrows], and disordered 3D glass spheres [(blue) $\triangle$ from [15]]. For reference, lines with slope $1 / 6$ (for the $p^{1 / 6}$ law) and 1/4 (sometimes quoted as effective exponent for low pressures) are shown.

est scale. On shorter scales, the material appears somewhat stiffer: As discussed in Appendix B, numerical measurements of the elastic modulus $C_{22}$ can be performed on various length scales, the shorter ones, in the case when displacements are locally controlled, leading to larger apparent values of $C_{22}$. In addition, we shall show in Sec. IV E that there is a strong contribution from non-plane-wave modes which cannot be expected to be described by continuum elasticity.

It might therefore be concluded that a simple long wavelength description gives a good first approximation of the propagation velocity of the coherent wave front, but that modes that are not accurately described by a long wavelength approximation contribute substantially to the wave propagation for the system sizes and excitation method employed here. For a triangular lattice of monodisperse balls, we compare the analytical expressions for the sound speed Eq. (18) and (19), which are derived in Sec. V for infinitely large lattices, with simulations on finite lattices. Both the frictionless and frictional cases are in excellent agreement, even though the frictional one shows appreciable finite-size corrections. The simulation for $2 \mathrm{D}$ disordered frictional case shows results very similar to the triangular lattice-including the $p^{1 / 6}$ scaling expected naively from the Hertzian force law-for the range of pressures considered.

This quantitative agreement is somewhat surprising in view of the large difference in coordination numbers (6 vs barely larger than 3). This might partly be due to the small wavelength effects, which affect the results in disordered systems, while one easily observes the long wavelength result $c_{\mathrm{TOF}}=c_{\ell}$ with a perfect regular lattice.

The only 2D data we are aware of are for spheres on a triangular lattice. These systems are inevitably slightly polydisperse, which prevents the closing of all contacts between nearest neighbors on the lattice $[3,17,41]$, and in the limit of low pressure, the coordination number should not exceed 4 . However, once the reduced pressure is high enough for the elastic deflection of contacts to compensate for the open gaps, the behavior of the perfect lattice is retrieved. This effect can be evaluated with the reduced pressure defined in [41] as

TABLE III. The scaling exponents $\gamma$ and $\alpha$ for different granular systems.

\begin{tabular}{|c|c|c|}
\hline Granular system & $\gamma$ & $\alpha$ \\
\hline 1D chain or triang. latt., monodisperse ${ }^{\mathrm{a}}$ & $2 / 3$ & $1 / 3$ \\
\hline 1D chain or triang. latt., monodisperse ${ }^{b}$ & 1 & $1 / 3$ \\
\hline 1D chain, polydisperse ${ }^{\mathrm{a}}$ (numerical) & $\geqslant 2 / 3$ & $\geqslant 1 / 3$ \\
\hline 2D disordered ${ }^{\mathrm{a}}$ (numerical) & $\approx 1.5$ & $\approx 1$ \\
\hline
\end{tabular}

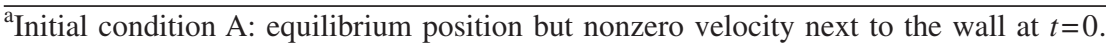

${ }^{\mathrm{b}}$ Initial condition B: zero velocity but nonzero displacement next to the wall at $t=0$. 


$$
P^{*}=\frac{3 P}{\alpha^{3 / 2} E^{*}}
$$

where $\alpha d$ is the width of the diameter distribution. Effects of polydispersity disappear as $P^{*}$ grows beyond 1 . For steel spheres the data of [17] (for which $\alpha \sim 10^{-4}$ ) fall close to our calculated values for the triangular lattice with friction when $p \geqslant 10^{-6}$, as expected. Even though there is a discrepancy in the velocity of the order of 10-20\%, this agreement is remarkable, since $c_{\mathrm{TOF}}$ has been calculated without any adjustable parameters. Possible finite-size effects might explain why these data lie below the theoretical frictional curve for the perfect lattice.

The triangular lattice of nylon balls [17] shows significantly larger rescaled velocity than expected. Possibly, this discrepancy is simply a reflection of the uncertainty in the effective elastic constant at the frequency range of the experiments: nylon is a viscoelastic material for which the Young modulus increases strongly with frequency. We do not know the values of the elastic constants at the experiment's frequencies, but nevertheless if we use a Young modulus twice as large as its zero frequency value (for the plot the zero frequency modulus was used), then the curve would shift as indicated by the arrows.

Finally, disordered 3D glass spheres [15] display smaller velocities than any $2 \mathrm{D}$ case. One possible explanation is that 2D experiments on planar sphere assemblies can be viewed, if we imagine stacking such layers on top of one another, as probing the stiffness or wave propagation along dense, well coordinated planes in a 3D material with extreme anisotropy. This renders plausible the observation of unusually high sound velocities, in comparison with ordinary 3D packings.

\section{E. Eigenfrequencies and eigenmodes}

In a rectangular sample of homogeneous elastic material with boundary conditions similar to those employed here, the eigenmodes are plane waves with wave vector $\mathbf{k}=\left(k_{1}, k_{2}\right)$ $=\left(n_{1} 2 \pi / L_{1}, n_{2} \pi / L_{2}\right)$. If the tensor of elastic moduli has the form given in (7), then it is straightforward to show that the associated frequencies $\omega_{+}, \omega_{-}$are given by $\omega_{ \pm}^{2}=\lambda_{ \pm} / \Phi_{3}^{*}, \lambda_{ \pm}$ being the eigenvalues of the acoustic tensor

$$
\mathbf{A}\left(k_{1}, k_{2}\right)=\left[\begin{array}{cc}
C_{11} k_{1}^{2}+C_{33} k_{2}^{2} & \left(C_{12}+C_{33}\right) k_{1} k_{2} \\
\left(C_{12}+C_{33}\right) k_{1} k_{2} & C_{33} k_{1}^{2}+C_{22} k_{2}^{2}
\end{array}\right],
$$

which implies that $\omega \propto k$ in the long wavelength limit.

We show the spectrum of eigenmodes for a granular packing of 600 grains at pressure $10^{-4}$ in Fig. 9(a), and a few selected eigenmodes in Fig. 10. There are a number of zero eigenvalues because of "rattler" grains not connected to the force network. The lowest nonzero modes correspond to (slightly distorted) solid body modes, which are similar to those expected from continuum theory. Remarkably, in the absence of friction it is much harder to identify eigenmodes corresponding to continuum media modes, even for low frequencies; and the low frequency modes are more abundant (not shown on the figures). Nevertheless, the transmission signal looks rather similar to the frictional case (Fig. 4).
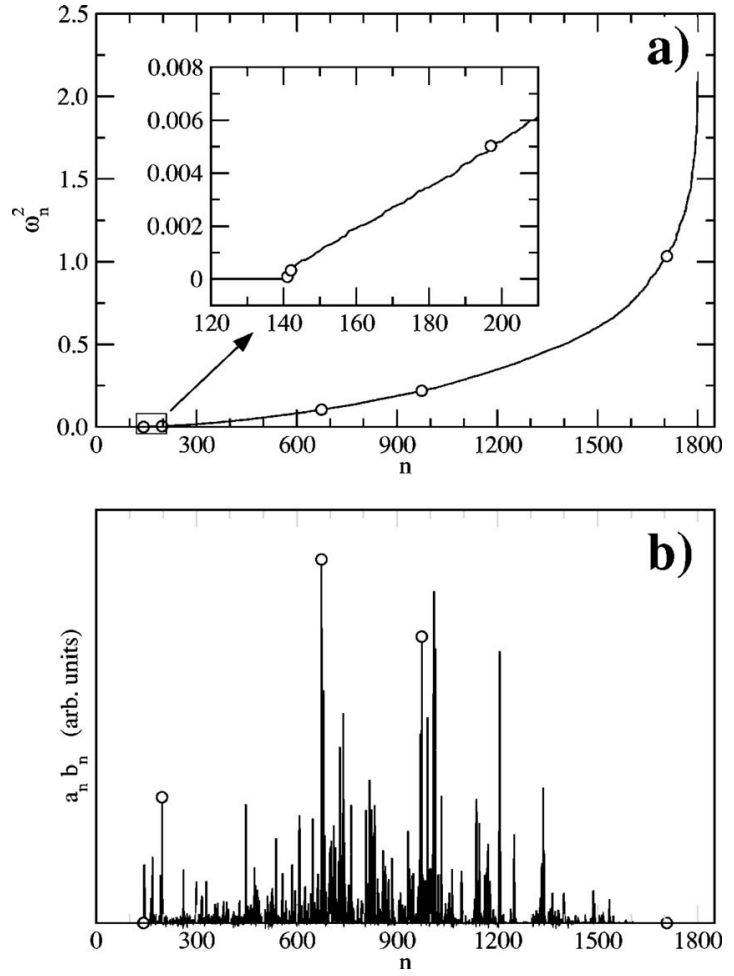

FIG. 9. (a) Eigenfrequencies of the linear system for the packing shown on Fig. 1. The squared eigenfrequencies are plotted against the number of the mode $n$. Modes $n=0, \ldots, 140$ have eigenvalue zero, as a consequence of rattler grains which are not connected to the network. The inset shows a magnification of the plot around the first few nonzero eigenvalues. (b) The contribution of the eigenmodes to the transmission signal $a_{n} b_{n}$ [see Eq. (3)]. On both panels the eigenmodes plotted on Fig. 10 are marked by circles.

There are a large number of localized eigenmodes [Fig. 10(f)], which do not contribute substantially to the signal transmission; clearly the modes that dominate the transmission are global modes [Fig. 9(b)]: they contain oscillating grains at both the source and the detector wall. But with the exception of only a few modes (with mode numbers 141-147 roughly), their appearance is quite different from simple plane waves [Fig. 10(c)-10(e)]. This indicates that, at least for the system sizes, pressures, and excitation method employed here, the transmission of sound cannot be captured completely by considering the material as a simple bulk elastic material. In fact, in the light of these findings it is remarkable how close the continuum prediction $c_{\ell}$ comes to $c_{\mathrm{TOF}}$. We will present a more extensive study on these eigenmodes elsewhere [42].

\section{F. The effects of damping}

Finally, we show here how damping affects the wave propagation. We added viscous dissipation to the Hertz contacts in the way described in Appendix A. The resulting system cannot be easily described by a linear model, and we obtained the wave propagation signal by molecular dynamics simulations of the grain oscillations. On Fig. 11 we show the transmission signal for a single configuration with various 

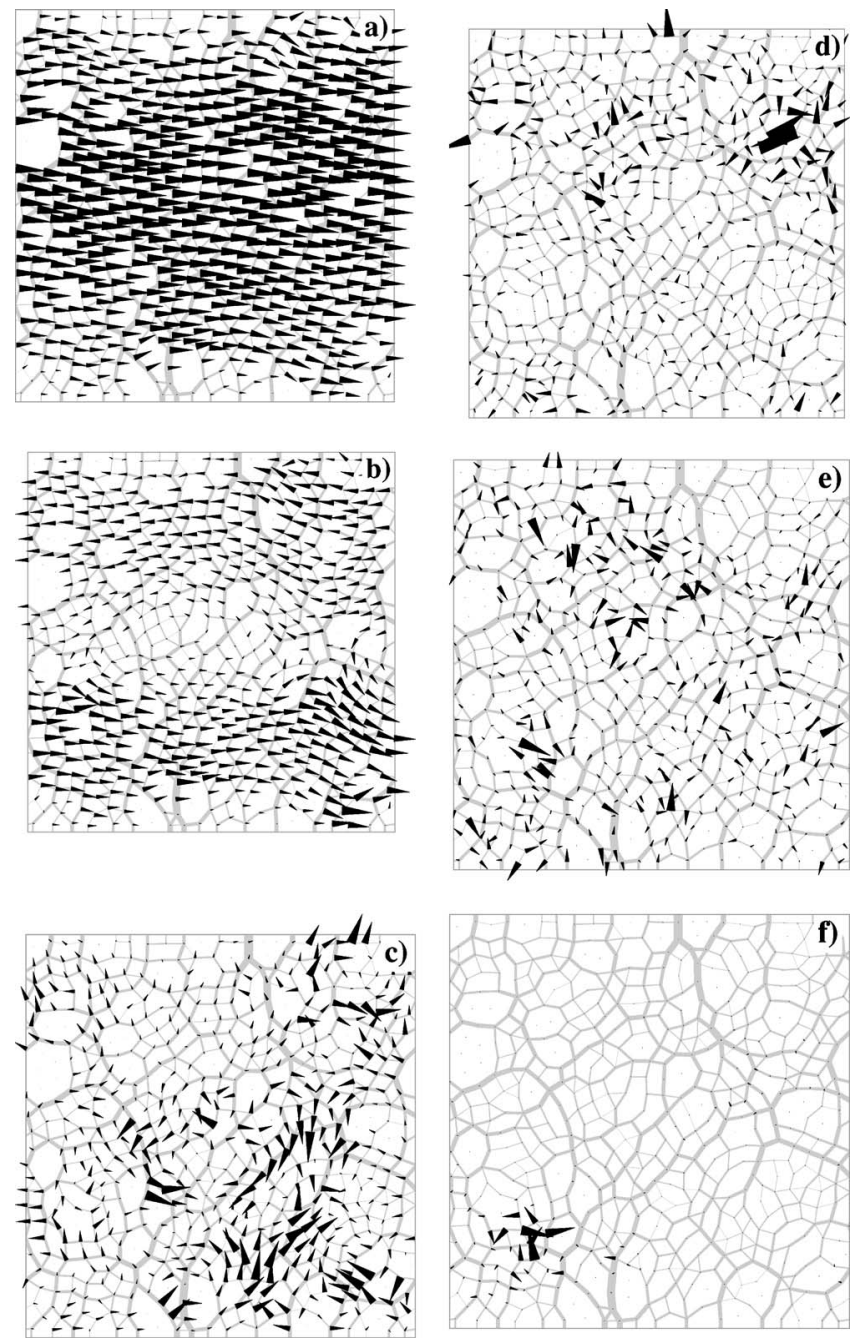

FIG. 10. A few selected eigenmodes of the linear system. (a) $n=141$ and (b) $n=142$ are the first two nonzero eigenmodes. They correspond to the lowest excitation modes of a continuum body, though slightly distorted by the disordered contact network. (c) $n$ $=197$, (d) $n=674$, and (e) $n=974$ are some of the modes that contribute significantly to the transmission of the signal. (f) $n=1707$ is a high frequency localized mode. The modes shown here are marked on the eigenvalue plot, Fig. 9.

levels of damping. For large damping the coherent part of the signal is only slightly altered, while the random part is strongly suppressed. This is in qualitative agreement with the experiments of Ref. [16], where damping was induced by adding a small amount of water to the glass bead packing.

\section{ANALYTIC RESULTS}

\section{A. 1D chain}

The problem of the propagation of a pulse in a 1D granular chain has been considered by many authors $[5,43-48]$ but the majority of the work is concentrated on analyzing an initially uncompressed chain. In this case the nonlinear force law plays an important role, as well as the fact that there are no restoring forces between the balls which initially just

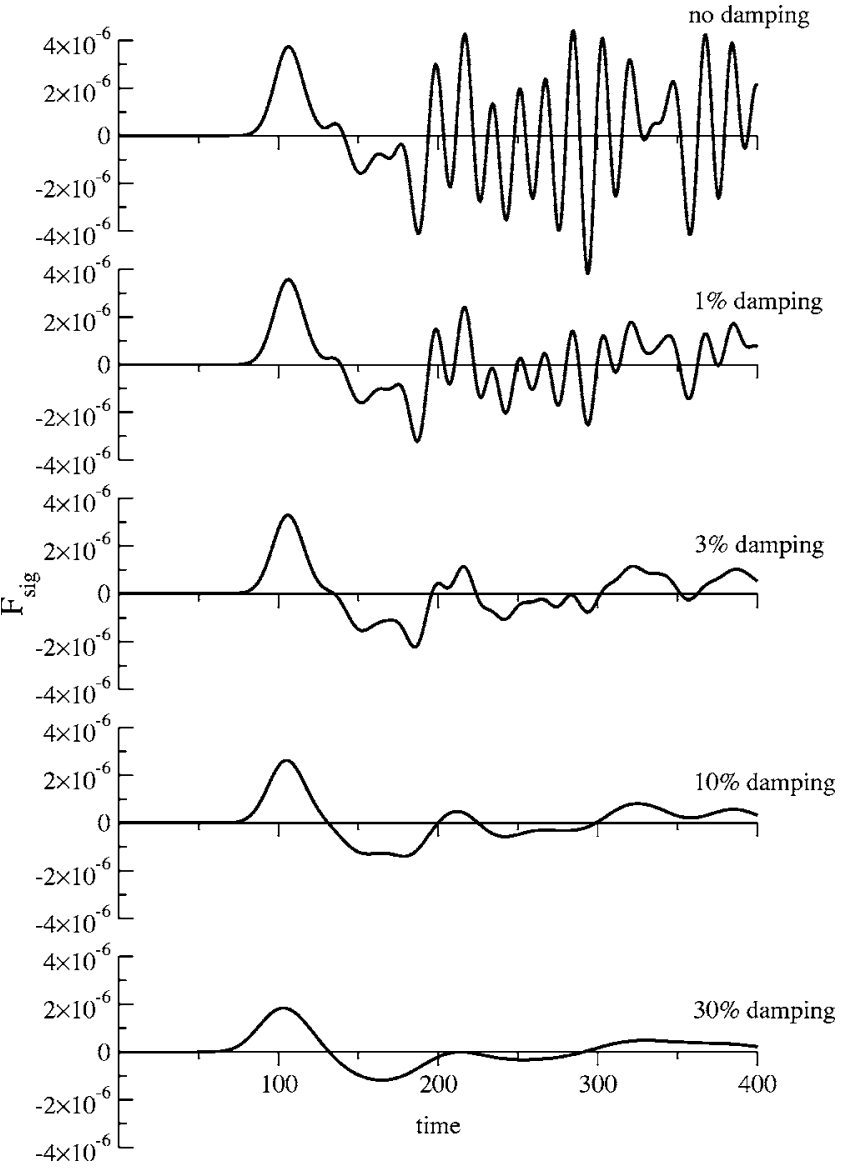

FIG. 11. The transmitted signal with damping. The level of damping is expressed as a fraction of the critical damping on each contact. The damping affects the coherent part of the signal much less than the random part.

touch. For comparison with sound propagation in granular media as a function of pressure, the relevant approach is to first linearize the equations of motion starting from a compressed chain, and then study the propagation of the pulse as governed by these linearized equations.

The simplest system resembling the problem of sound propagation in granular media (under pressure) is a $1 \mathrm{D}$ chain of identical elastic balls, confined and compressed between two walls. At $t=0$ we disturb the first ball (see below for details), this disturbance travels with sound speed $c$ in the chain, and arrives at the other wall at time $t_{0}=N \ell / c$, where $\ell$ is the diameter of the balls. For this system we can calculate the scaling exponents and the wave form analytically.

In Appendix $\mathrm{C}$ we calculate the attenuation exponent of this wave. For initial condition A, where the first ball has nonzero velocity but zero displacement at $t=0$, the force with which the last ball presses the wall at time $t_{0}$ scales with $N$ as

$$
F^{\mathrm{A}}\left(t_{0}\right) \sim N^{-2 / 3}
$$

Initial condition $\mathrm{B}$, where all balls start with zero velocity but the first has a finite displacement, gives a different answer: 


$$
F^{\mathrm{B}}\left(t_{0}\right) \sim N^{-1} .
$$

These are the attenuation exponents for a uniform 1D chain.

To derive the wave form analytically in the large system and long time limit, we consider the long wavelength expansion of the dispersion relation $(\mathrm{C} 2)$ :

$$
\omega_{n} \approx c k_{n}-\frac{c \ell^{2}}{24} k_{n}^{3}
$$

A propagating wave solution $u(x, t)=A \exp (i k x-\omega t)$, where for long wavelengths $x$ can be considered continuous, has to satisfy the following partial differential equation to match dispersion relation (11):

$$
-\frac{\partial u}{\partial t}=c \frac{\partial u}{\partial x}+\frac{c \ell^{2}}{24} \frac{\partial^{3} u}{\partial x^{3}}
$$

Changing variables to the comoving frame, $\xi=x-c t$, the $\partial u / \partial x$ term drops out. Looking at similarity solutions of the form

$$
u(\xi, t) \sim t^{-g} U\left(w=\frac{\xi}{t^{\alpha}}\right),
$$

we obtain $\alpha=1 / 3$ and

$$
0=-g U(w)-\frac{w}{3} U^{\prime}(w)+\frac{c \ell^{2}}{24} U^{\prime \prime \prime}(w) .
$$

This leads to different classes of solutions for different attenuation exponents $g$. First we consider the case $g=0$, which leads to Airy functions: $U_{0}^{\prime}(w)=\operatorname{Ai}\left(2 w /\left(c \ell^{2}\right)^{1 / 3}\right)$. We can generate further solutions by differentiating Eq. (14). For example, by differentiating once and twice we find that $U$ $=U_{0}^{\prime}$ solves Eq. (14) for $g=1 / 3$, while $U_{0}^{\prime \prime}$ solves it for $g$ $=2 / 3$. For $u(x, t)$ this gives solutions, e.g., for $g=1 / 3$ as $t^{-1 / 3} \operatorname{Ai}\left(2(x-c t) /\left(c \ell^{2} t\right)^{1 / 3}\right)$, which as we show soon is the selected solution for initial condition A. At this point we need more information to see which solution is selected: Eqs. (9) and (10) tell the exponent of the scaling of the force at the wall with $N$. Note that $u(x, t)$, is the propagating solution in a semi-infinite medium; the solution for a reflecting wall boundary condition, $u(x=(N+1) \ell, t)=0$, is composed of two counterpropagating waves. Now the force at the wall is proportional to the displacement of the ball (at $x=N \ell$ ) next to the reflecting wall [at $x=(N+1) \ell]$; hence it can be written as

$$
F(t)=K\{u(x, t)-u(2(N+1) \ell-x, t)\},
$$

which with $x=N \ell$ and $t=t_{0}+\tau$ gives for the $g=1 / 3$ solution

$$
\begin{gathered}
F\left(t_{0}+\tau\right) \sim K \ell\left(\frac{c t}{\ell}\right)^{-1 / 3}\left\{\operatorname{Ai}\left(\frac{-2 c \tau}{\left(c \ell^{2} t\right)^{1 / 3}}\right)-\operatorname{Ai}\left(\frac{4 \ell-2 c \tau}{\left(c \ell^{2} t\right)^{1 / 3}}\right)\right\} \\
\sim-4 K \ell\left(N+\frac{c \tau}{\ell}\right)^{-2 / 3} \mathrm{Ai}^{\prime}\left(\frac{-2 c \tau / \ell}{(N+c \tau / \ell)^{1 / 3}}\right) .
\end{gathered}
$$

Note that because of the extra differentiation the decay exponent of the force on $N$ does not equal $g=1 / 3$ but instead becomes $\gamma=g+1 / 3=2 / 3$. This scaling exponent is the same

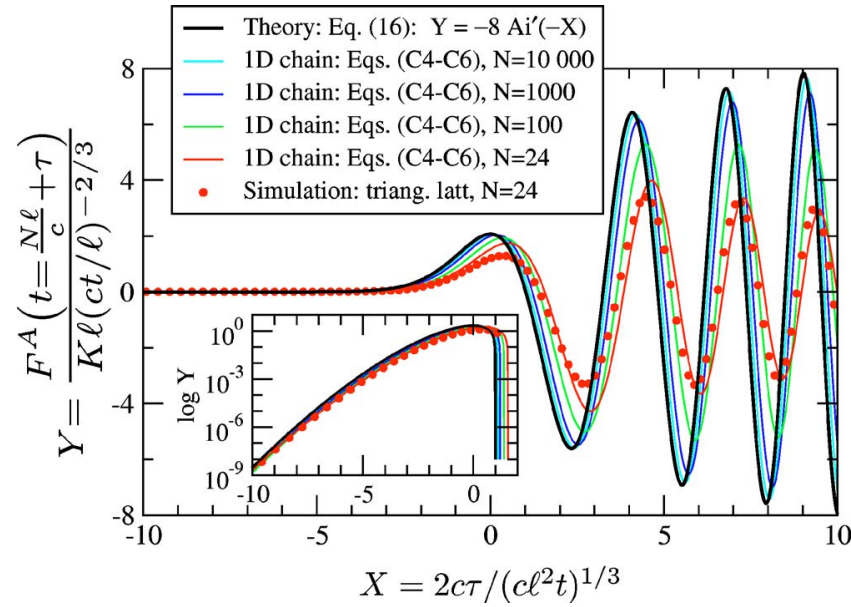

FIG. 12. (Color online) Comparison of signal shapes for initial condition A. The theoretical prediction of Eq. (16) (thick black line) is compared to the $1 \mathrm{D}$ chain of identical balls, numerical sum in Eqs. (C4)-(C6) [color lines]. The simulation of a perfect triangular lattice [(red) full circles] is very close to the 1D chain of the same size. Note that Eq. (16) has an undetermined multiplicative factor as it is obtained as a solution of a linear equation.

as for the initial condition A in Eq. (9), showing that indeed the $g=1 / 3$ solution is selected here.

To match the initial condition B, we need to use the $g$ $=2 / 3$ solution

$$
F^{\mathrm{B}}\left(t_{0}+\tau\right) \sim-4 K \ell\left(N+\frac{c \tau}{\ell}\right)^{-1} \mathrm{Ai}^{\prime \prime}\left(\frac{-2 c \tau / \ell}{(N+c \tau / \ell)^{1 / 3}}\right) .
$$

The solution of the $1 \mathrm{D}$ chain obtained by numerically evaluating the sum (C4) converges for $N \rightarrow \infty$ to the analytical solution; see Fig. 12 where the initial condition A is plotted.

At this point we can understand the connection between the two initial conditions. Initial condition B is related to A by a time differentiation. Since the equations are linear, the solutions are similarly related to each other. The above solutions have the structure that differentiating one of them and dropping subdominant terms gives another of the solutions, with exponent $g$ which has increased by $1 / 3$.

If we allow for disorder in the 1D chain, the results change slightly. We introduce disorder by varying the radii of the ball, and solve this 1D system numerically, as described in Sec. III for 2D and 3D packings. On Fig. 7 we show the exponents of the polydisperse 1D chain. Both the amplitude exponent $\gamma$ and the width exponent $\alpha$ appear to be larger than in the case of identical balls, but the results are not clear enough to extract a value for the exponents.

The above analysis shows that in the long time limit, the propagation of an initially localized pulse is governed by an Airy equation - as Fig. 12 shows, the first pulse and the first oscillations behind it converge to an Airy function type behavior when viewed in a frame comoving with the initial pulse. Note that the kinetic energy in the leading pulse decays rapidly as $t^{-2 g-1 / 3}$ : 


$$
\begin{aligned}
E_{\text {kin }} & =\sum_{\text {init pulse }} \frac{m}{2} \dot{u}_{n}^{2}(t) \simeq \frac{m}{2} \sum_{\text {init pulse }} c^{2} \frac{t^{-2 g}}{t^{2 / 3}}\left[U^{\prime}\left(w_{n}\right)\right]^{2} \sim \frac{t^{-2 g}}{t^{2 / 3}} t^{1 / 3} \\
& \sim t^{-2 g-1 / 3},
\end{aligned}
$$

because the number of terms in the sum which contribute to the first peak is proportional to the width of the pulse, which scales as $t^{1 / 3}$. Hence for the pulse shown in Fig. 12, the kinetic energy in the first pulse decays as $t^{-1}$, since $g=1 / 3$. This illustrates that as time progresses, more and more of the energy is stored in the region behind the first pulse. The oscillations in this region are relatively incoherent, with a frequency comparable to the maximum frequency of the dispersion relation. As the size of this region increases linearly with time, the typical amplitude of these oscillations decays as $t^{-1 / 2}$. One can also obtain a $t^{-1 / 2}$ type decay directly from a steepest descent analysis near the maximum of the dispersion relation of the linearized equations of motion.

The fact that the first pulse in 1D chains is described by an Airy function has been noted before $[44,47]$. Most of these studies are for initially uncompressed chains, however. In this case, all the energy remains confined in the first pulse, due to the absence of restoring forces. As a result, the exponent of the time dependence of the amplitude is different, and consistent with energy conservation in the leading pulse.

\section{B. Triangular lattice}

In view of the experiments of Gilles and Coste [17], it is illuminating to also apply these results to the simplest $2 \mathrm{D}$ system: a triangular lattice of balls, with rectangular boundaries. The initial condition is given on balls touching one wall of the rectangle, and we assume that a lattice direction of the triangular lattice is parallel to this wall. The longitudinal sound speed in a perfect triangular lattice (no polydispersity) of Hertzian balls can be easily calculated; see, e.g., [3]. For the frictionless and frictional case, respectively, it is given by

$$
\begin{gathered}
\frac{c^{\text {no fric }}}{\sqrt{E^{*} / \rho}}=\frac{3^{19 / 12}}{2^{3 / 2} \pi^{1 / 2}}\left(\frac{p}{E^{*}}\right)^{1 / 6}, \\
\frac{c^{\text {fric }}}{\sqrt{E^{*} / \rho}}=\frac{3^{19 / 12}}{2^{3 / 2} \pi^{1 / 2}} \sqrt{1+\frac{\eta}{3}}\left(\frac{p}{E^{*}}\right)^{1 / 6},
\end{gathered}
$$

where the parameter $\eta$ is the ratio of the tangential and radial stiffnesses of a Hertz-Mindlin contact [see Eq. (A4)].

One way to calculate this is to map to the 1D chain of identical balls. If in the triangular lattice the longitudinal motion is perpendicular to rows, then a row of $M$ balls moves together, corresponding to a single ball in 1D. Thus of the $N$ rows each has mass $m_{\text {eff }}=M \rho \pi / 6$, they are separated by distance $\ell=\sqrt{3} / 2$ (recall our length unit was the ball diameter), and connected by an effective spring $K_{\text {eff }}$ $=3^{7 / 6} 2^{-2} M p^{1 / 3}$. In the frictional case $K_{\text {eff }}$ has an additional factor of $(1+\eta / 3)$.

This way we also predict the shape of the signal for the triangular lattice (see Fig. 12). The cause of the slight deviation from the 1D chain result is a consequence of the fact that in the triangular lattice the springs connecting to the walls are different: $K_{\text {wall }} / K_{\text {bulk }}=3^{5 / 6} / 4 \approx 0.62$.

If the radii of the balls are polydisperse, then at pressures low enough (that the length scale of the elastic deformations become comparable to the polydispersity) the stress field fluctuates spatially. The effect of this on the sound speed has been calculated by Velický and Caroli [3] in a mean-field approximation.

\section{DISCUSSION}

We presented numerical simulations of pulse propagation in $1 \mathrm{D}, 2 \mathrm{D}$, and $3 \mathrm{D}$ granular systems. This response can be decomposed into an initial coherent part, which is independent on the details of the packing, and a subsequent random part, which is strongly realization dependent. We have focused on the properties of the initial coherent front. Our first observation is that the response to a pulse propagates linearly in time, defining a time-of-flight velocity, and does not follow force chains.

The fact that the packings in our numerical simulations have roughly the same number of grains per container side (although in 2D) as the systems which have been studied experimentally by Jia et al. [15], and that our temporal signals are very comparable to the experimental ones, makes us confident that our simulation results can be fruitfully compared to experiments like these. Indeed we find that the 3D experimental and 2D numerical results for the time-of-flight velocity are in reasonably good agreement. The experiments in 2D are done on triangular lattices, and we also study numerically and analytically pulse propagation on such lattices with and without friction. The experiments for steel spheres and the predictions for frictional lattices are in good agreement (even though there are some subtle points regarding the scaling with pressure; see below).

We also compare our numerical results for the disordered system with predictions following from numerical estimates of the effective long wavelength elastic constants of our packings. Remarkably, even though elastic constants predict the time-of-flight velocity reasonably well, there is a $40 \%$ discrepancy between the predicted and observed velocities for our systems. A possible reason for this is that our pulses may probe the system on short scales which are not governed by a long wavelength expansion; indeed a (preliminary) analysis of the spatial structure of the modes that contribute significantly to pulse propagation indicates that most modes appear rather different from simple plane waves. It is likely, but very hard to check numerically (at least with the methods used in this paper), that for propagation over larger distances (such as those probed in the engineering literature) the elastic approximation becomes better, and the dominant modes would become simple plane waves. The crucial open question becomes thus what sets the length scale at which such description becomes applicable. Recently this issue has also emerged within the context of the proposal that the static behavior of granular packings of hard particles is governed by a critical point ("point J") in a jamming phase diagram [8]. We will come back to the relation to the jamming phase diagram elsewhere [42]. 
We also found that the amplitude of the coherent part and its width scales with a power of the distance as the signal propagates. For the initial condition where grains touching one wall have nonzero velocity, the amplitude exponent is $\gamma \approx 1.5$ for disordered 2D systems, while it is $2 / 3$ (exact result) for a $1 \mathrm{D}$ chain of identical balls. The exponent of the signal width is $\alpha \approx 1$ for the disordered 2D system, and 1/3 for the $1 \mathrm{D}$ chain. The shape of the signal can be computed as well, and it is given by Airy functions for the 1D chain. A triangular lattice of identical balls can be mapped (except for the strength of the wall springs) to the 1D chain, predicting the same exponents and signal shape.

A final issue that we studied in detail is the variation of the sound velocity with pressure, since this is an important experimental parameter. Our simulations for frictional contacts recover the expected $p^{1 / 6}$ behavior for the time-of-flight velocity and bulk modulus, but not for the shear modulus: we found that the transversal wave speed scales approximately as $p^{1 / 4}$. These results should be compared to results for frictionless sphere packings with Hertzian contacts as studied by O'Hern et al. [8]. They found that the bulk modulus $B$ $\sim p^{1 / 3}$ at low pressure, while the shear modulus $G$ scaled as $p^{2 / 3}$, resulting in $c_{\ell}=\sqrt{[B+(4 / 3) G] / \rho} \sim p^{1 / 6}$ and $c_{\mathrm{t}}=\sqrt{G / \rho}$ $\sim p^{1 / 3}$.

Some of the experimental data for $c_{\mathrm{TOF}}[15-17]$ or for resonance frequencies [49] in bead assemblies, and some evaluations of elastic moduli in numerical simulations [2,41], evidence a larger exponent, or at least some departure from the $p^{1 / 6}$ scaling. The physical origin of such observations has been the subject of considerable debate [2-4,41]. In fact, results for the pressure dependence of sound velocity in disordered glass bead packings are somewhat different according to the conditions of the experiment, and apparent values of exponents $\alpha$ in a $c \sim p^{\alpha}$ fit vary roughly between 0.16 and 0.25 . This calls for detailed investigations of the influence of the internal state of packings on sound velocities and their pressure dependence. While the data published in [15], shown on Fig. 8, indicate a crossover from $p^{1 / 4}$ at low $p$ to $p^{1 / 6}$ at higher pressure, Domenico's results [50], corresponding to much larger confining stress, are fitted by a $p^{1 / 4}$ law. Other data by Jia and Mills (see, e.g., Ref. [16], Fig. 10) agree with $c \sim p^{0.21}$ on the whole studied pressure range, while Sharifipour et al. [25] report in some cases exponents $\alpha$ as high as 0.28 . Pressure dependences with exponents $\alpha$ $\sim 0.25$ often observed with sands (see, e.g., [19]) are likely to be related to the non-Hertzian behavior of contacts between angular particles (or between asperities of rough particles) as discussed by Goddard [4], and are outside the scope of our simulations.

Another suggested origin for a different effective scaling for Hertzian contacts is the increase of coordination number with pressure $[2,4,8,17,41]$, which gradually stiffens the packing. In our case, this increase is rather small (from $z^{*}$ $\simeq 3.2$ to $z^{*} \simeq 3.5$ ) and does not entail any deviation from the $p^{1 / 6}$ scaling for the effective longitudinal speed $c_{\text {TOF }}$.

Such an explanation by pressure-induced recruitment of additional contacts seems more plausible in regular lattices of nominally identical spheres $[3,17,41,49]$, in which a slight polydispersity (or lack of sphericity) causes lattice imperfections and strongly reduces the coordination number, which only recovers the perfect lattice value at high enough confining pressure, $P^{*} \geqslant 1$ (see Sec. IV D). We can expect such a mechanism to explain the experimental observations by Duffy and Mindlin [49] and Gilles and Coste [17], as numerical studies of elastic moduli [41], as well as a selfconsistent effective medium approach by Velický and Caroli [3], both find deviations from a $p^{1 / 6}$ scaling of long wavelength sound. This effect is of course absent in our simulations of perfect lattices.

\section{ACKNOWLEDGMENTS}

This research has been supported by the Dutch FOM Foundation and the PHYNECS training network of the European Commission under Contract No. HPRN-CT-200200312. M.v.H. acknowledges support from NWO. Laboratoire des Matériaux et des Structures du Génie Civil is a joint laboratory, depending on Laboratoire Central des Ponts et Chaussées, Ecole Nationale des Ponts et Chaussées, and Centre National de la Recherche Scientifique. We thank Christophe Coste, Martin Depken, Chay Goldenberg, Ray Goldstein, Xiaoping Jia, Bert Peletier, Adriana Pesci, and Tamás Unger for discussions.

\section{APPENDIX A: CONTACT FORCES IN THE NUMERICAL MODEL}

The normal force between two particles in contact is given by the 3D Hertz law [51], which is the force between two elastic spheres (labeled 1 and 2)

$$
F_{n}=\frac{4}{3} \sqrt{R_{12}} E_{12}^{*} n^{3 / 2},
$$

where the effective radius $R_{12}=\left[\left(R_{1}\right)^{-1}+\left(R_{2}\right)^{-1}\right]^{-1}$ and effective Young modulus $E_{12}^{*}=\left[\left(E_{1}^{*}\right)^{-1}+\left(E_{2}^{*}\right)^{-1}\right]^{-1}$ are half of the harmonic averages of the two grain's parameters. Here we introduced the material parameter $E^{*}=E /\left(1-\nu^{2}\right)$ (modified Young modulus, nonstandard notation), where $E$ is the Young modulus and $\nu$ is the Poisson number. The distance of approach (or "virtual normal overlap") is given by $n=R_{1}$ $+R_{2}-r_{12}$, where $r_{12}$ is the distance of the two particle centers. Grain-wall interaction can be obtained by setting $R_{\text {wall }}$ $=\infty$, and we used hard walls $\left(E_{\text {wall }}=\infty\right)$.

Implementing the frictional force is less straightforward, because frictional contacts can have a memory of their history. The standard approach is to consider changes in the tangential force with Mindlin's approximation [51]:

$$
\Delta F_{t}=8 G_{12}^{*} \sqrt{R_{12} n} \Delta t
$$

where the elastic constant $G_{12}^{*}=\left[\left(G_{1}^{*}\right)^{-1}+\left(G_{2}^{*}\right)^{-1}\right]^{-1}$ can be calculated from the two grains' material parameters $G^{*}$ $=E /[2(1+\nu)(2-\nu)]=E^{*}(1-\nu) /(4-2 \nu)$, and the virtual tangential displacement $t$ of the particle surfaces is determined from their centers' motion and their rotations. This incremental force law is augmented with the Coulomb condition

$$
\left|F_{t}\right| \leqslant \mu F_{n}
$$

where we take the friction coefficient $\mu$ as parameter. It is interesting to note that for a given contact the ratio of the 
TABLE IV. Ratio of apparent elastic moduli deduced from Eq. (B2) for $n=1$ and 2 to the global values.

\begin{tabular}{ccccc}
\hline \hline$p$ & $\widetilde{C}_{22}(1) / C_{22}$ & $\widetilde{C}_{22}(2) / C_{22}$ & $\widetilde{C}_{33}(1) / C_{33}$ & $\widetilde{C}_{33}(2) / C_{33}$ \\
\hline $10^{-7}$ & $0.85 \pm 0.05$ & $0.74 \pm 0.09$ & $0.81 \pm 0.14$ & $1.01 \pm 0.24$ \\
$10^{-6}$ & $0.88 \pm 0.04$ & $0.80 \pm 0.06$ & $0.90 \pm 0.20$ & $0.96 \pm 0.16$ \\
$10^{-5}$ & $0.91 \pm 0.05$ & $0.86 \pm 0.06$ & $0.85 \pm 0.10$ & $0.89 \pm 0.11$ \\
$10^{-4}$ & $0.93 \pm 0.04$ & $0.88 \pm 0.04$ & $0.85 \pm 0.10$ & $0.90 \pm 0.07$ \\
\hline \hline
\end{tabular}

normal stiffness to the tangential stiffness (despite the very different force laws) is constant; we call it $\eta$ :

$$
\eta:=\frac{d F_{t} / d t}{d F_{n} / d n}=\frac{8 G^{*}}{2 E^{*}}=1-\frac{\nu}{2-\nu},
$$

assuming the two grains have the same elastic parameters. For example, for $\nu=0$, the value we use in most of the simulations, the two effective stiffnesses $d F_{t} / d t$ and $d F_{n} / d n$ are equal, so for vibrations neither the radial nor the frictional part of the contact will dominate the other. [Note that Eq. (A4), a consequence of approximation (A2), is really only valid for $\left|F_{t}\right| \ll \mu F_{n}$, as pointed out in [3].]

The Coulomb condition introduces dissipation, because contact surfaces may slip at nonzero force. The ensuing dissipation occurs only when the yielding threshold is exceeded, and not in the infinitesimal amplitude oscillations we study here. Nevertheless, in some cases we wish to add dissipation, for example, when creating the packing from the granular gas, or when studying the effect of damping on the small amplitude oscillations. For this purpose we chose a particular form of damping, which is at every instant a given constant fraction of the linear critical viscous damping, both for the normal and for the tangential force. Through this procedure, the effective damping force, like the normal and tangential Hertz-Mindlin forces, depends nonlinearly on the distance of approach $n$. We impose that the total radial force, which now also contains the viscous contribution, never becomes attractive.

This choice is appealing theoretically because it contains only one nondimensional parameter to control the strength of the dissipation. In practice it is not clear what the best approximation is of the real (dry or wet grains') dissipation. In any case there should be some contacts that dominate the dissipation. For those contacts the viscous force is a certain fraction of the critical damping. Our approach is that we impose this ratio on all contacts.

\section{APPENDIX B: SMALL SCALE STATIC ELASTICITY}

Let us consider a homogeneous macroscopic sample of an elastic material with the same symmetries and boundary conditions as our numerical systems, and apply a body force (per unit volume):

$$
f_{\alpha}^{(n)}(y)=f_{0} \sin \left(\frac{n \pi y}{L_{2}}\right),
$$

depending on coordinate $y$, and directed parallel to axis $\alpha$ (1 or 2). With boundary conditions $\mathbf{u}=0$ on the top $\left(y=L_{2}\right)$ and bottom $(y=0)$ walls, and lateral periodicity $\left(L_{1}\right.$ is the system width) the corresponding displacement field only has a nonvanishing coordinate $u_{\alpha}$, given by

$$
u_{\alpha}^{(n)}(y)=\frac{f_{0} L_{2}^{2}}{n^{2} \pi^{2} C_{\alpha}} \sin \left(\frac{n \pi y}{L_{2}}\right),
$$

with $C_{\alpha}=C_{22}$ for $\alpha=2$ and $C_{\alpha}=C_{33}$ for $\alpha=1$. Hence the total elastic energy

$$
\tilde{W}_{\alpha}^{(n)}=\frac{L_{1} L_{2}^{3} f_{0}^{2}}{4 n^{2} \pi^{2} C_{\alpha}} .
$$

To mimic the force field of Eq. (B1) in our discrete samples, each nonrattler bead $i$ is submitted to a force

$$
F_{i, \alpha}^{(n)}=\frac{4 \pi R_{i}^{3}}{3 \Phi_{3}^{*}} f_{0} \sin \left(\frac{n \pi y_{i}}{L_{2}}\right),
$$

while the bottom and top walls are fixed and an apparent elastic modulus is obtained from the total elastic energy using Eq. (B2). The resulting "local" constants, denoted as $\widetilde{C}_{22}(n)$ and $\widetilde{C}_{33}(n)$ are compared in Table IV, for $n=1$ and 2 , to the corresponding "global" values (given in Table II). The longitudinal constant $C_{22}$, measured in this way, is only slightly lower, but roughly agrees with the previous result. Results for transverse constants are similar, except that sample to sample fluctuations are somewhat larger. In view of the small sample size, and the importance of boundary effects, it can be concluded, especially for the higher pressures, that the static elastic response to force fields is in reasonable agreement with the equations of elasticity involving the moduli measured on globally deforming the sample. This is further confirmed by another set of static response calculations, in which displacements rather than forces are imposed. Let us define regularly spaced horizontal lines through the sample at $y=k L_{2} /(n+1)$ for $0 \leqslant k \leqslant n+1$ (so that $k=0$ corresponds to the bottom and $k=n+1$ corresponds to the top), with $n$ an odd number. Let us impose constant displacements $u=0$ in direction $\alpha$ on line $k$ if $k$ is even, $u=(-1)^{l} u_{0}$ on line $k$ if $k=2 l+1$ is odd. In a homogeneous elastic system, the displacement field varies linearly between neighboring lines $k$ and $k+1$, and the total elastic energy reads

$$
\check{W}_{\alpha}^{(n)}=\frac{(n+1)^{2} L_{1}}{2 L_{2}} C_{\alpha} u_{0}^{2} .
$$

Imposing on the center of each particle crossed by the $k$ lines the displacements of the same point in a homogeneous con- 
TABLE V. Ratio of apparent elastic moduli deduced from Eq. (B3) for $n=1$ and 3 to the global values.

\begin{tabular}{ccccc}
\hline \hline$p$ & $\check{C}_{22}(1) / C_{22}$ & $\check{C}_{22}(3) / C_{22}$ & $\check{C}_{33}(1) / C_{33}$ & $\check{C}_{33}(3) / C_{33}$ \\
\hline $10^{-7}$ & $1.01 \pm 0.02$ & $1.09 \pm 0.03$ & $1.26 \pm 0.16$ & $2.49 \pm 0.69$ \\
$10^{-6}$ & $1.01 \pm 0.01$ & $1.07 \pm 0.07$ & $1.28 \pm 0.20$ & $2.05 \pm 0.45$ \\
$10^{-5}$ & $1.01 \pm 0.02$ & $1.06 \pm 0.03$ & $1.15 \pm 0.11$ & $1.65 \pm 0.30$ \\
$10^{-4}$ & $1.00 \pm 0.01$ & $1.03 \pm 0.02$ & $1.08 \pm 0.05$ & $1.37 \pm 0.14$ \\
\hline
\end{tabular}

tinuum (and leaving the rotations free), computing the total elastic energy, and using formula (B3), one deduces other values for $C_{22}$ and $C_{33}$, denoted as $\widetilde{C}_{\alpha}(n)$, given in Table $\mathrm{V}$ for $n=1$ and 3 . Once again, they are fairly close to the global moduli of Table II, especially for the higher $p$ values, albeit slightly larger. We therefore conclude that the elastic moduli are only weakly affected by finite-size effects.

\section{APPENDIX C: 1D CHAIN OF IDENTICAL BALLS}

We model the 1D chain of identical balls with $N$ identical particles of mass $m$, separated by distance $\ell$, and connected by linear springs of stiffness $K$. The first and last balls are also connected with identical springs to walls. This system models small amplitude oscillations of Hertzian balls under finite static pressure. The $n$th eigenmode of this simple linear system is given by

$$
u_{x}^{(n)}(t)=\sin \left(k_{n} x\right) \sin \left(\omega_{n} t+\phi_{n}\right)
$$

where $u(t)$ is the displacement of a ball, and we label the balls by their position $x=i \ell$, where $i=1,2, \ldots, N$. The wave numbers and eigenfrequencies are

$$
k_{n}=\frac{n \pi}{(N+1) \ell}, \quad \omega_{n}=2 \sqrt{\frac{K}{m}} \sin \frac{k_{n} \ell}{2} .
$$

The above dispersion relation determines the longitudinal sound speed

$$
c=\left.\frac{d \omega}{d k}\right|_{k=0}=\ell \sqrt{\frac{K}{m}}
$$

by which the long wavelength waves propagate. The full solution is given by

$$
u_{x}(t)=\sum_{n=1}^{N} a_{n} \sin \left(k_{n} x\right) \sin \left(\omega_{n} t+\phi_{n}\right),
$$

where the amplitudes $a_{n}$ are computed by projecting the initial condition onto the modes. The two cases considered here are (A) $\dot{u}_{1}(t=0)=c$, and (B) $u_{1}(t=0)=\ell$; all other displacements and velocities at $t=0$ are zero. This gives

$$
a_{n}^{\mathrm{A}}=\frac{2 \ell}{N+1} \cos \frac{k_{n} \ell}{2}, \quad a_{n}^{\mathrm{B}}=\frac{2 \ell}{N+1} \sin k_{n} \ell .
$$

We are interested in the time dependence of the force with which the $N$ th ball presses the wall:

$$
F(t)=K u_{N}(t)
$$

We cannot calculate this in closed form, but can for example look at its value at the time "a wave would arrive": $t_{0}$ $=N \ell / c$. Substituting the first initial conditions $a^{\mathrm{A}}$ into Eq. (C4) and rewriting the highly oscillating terms we get

$$
\begin{aligned}
F^{\mathrm{A}}\left(t_{0}\right)= & \sum_{n=1}^{N} \frac{2 K \ell}{N+1} \cos \frac{k_{n} \ell}{2} \sin k_{n} \ell \\
& \times \times \sin \left(k_{n} \ell+\frac{k_{n}^{3}}{k_{0}^{3}}+\frac{O\left(k_{n}^{5} \ell^{2}\right)}{k_{0}^{3}}\right)
\end{aligned}
$$

where $k_{0}=(24 / N)^{1 / 3} / \ell$. The terms in the sum become highly oscillating for $k_{n} \gtrsim k_{0}$, effectively canceling each other. The dominant contribution therefore comes from terms with $k_{n}$ $<k_{0}$ (or equivalently $k_{n}<$ const $\times k_{0}$, as ultimately we will only compute scaling exponents). The sum of the slowly varying terms is approximated by the integral

$$
\begin{aligned}
F^{\mathrm{A}}\left(t_{0}\right) \sim & \int_{0}^{k_{0}} \frac{2 K \ell d k}{\pi} \cos \frac{k \ell}{2} \sin k \ell \\
& \times \times \sin \left(k \ell+\frac{k^{3}}{k_{0}^{3}}+\frac{O\left(k^{5} \ell^{2}\right)}{k_{0}^{3}}\right) .
\end{aligned}
$$

We look at the asymptotics $N \rightarrow \infty$ (implying $k_{0} \rightarrow 0$ ), for which we have to find the terms lowest order in $k_{0}$. This yields

$$
F^{\mathrm{A}}\left(t_{0}\right) \sim k_{0}^{2} .
$$

Interestingly the second initial condition gives a different answer:

$$
F^{\mathrm{B}}\left(t_{0}\right) \sim k_{0}^{3}
$$

The above two relations immediately lead to Eqs. (9) and (10). 
[1] D. W. Howell, R. P. Behringer, and C. T. Veje, Chaos 9, 559 (1999).

[2] H. A. Makse, N. Gland, D. L. Johnson, and L. M. Schwartz, Phys. Rev. Lett. 83, 5070 (1999).

[3] B. Velicky and C. Caroli, Phys. Rev. E 65, 021307 (2002).

[4] J. D. Goddard, Proc. R. Soc. London, Ser. A 430, 105 (1990).

[5] C. Coste, E. Falcon, and S. Fauve, Phys. Rev. E 56, 6104 (1997).

[6] Ultrasound is the word typically used for sound at frequencies higher than the upper frequency of the human ear, i.e., higher than about $20 \mathrm{kHz}$. In the experiments to which we will compare, the typical frequency is of order $0.5 \mathrm{MHz}$.

[7] J. Astrom, M. Kellomaki, M. Alava, and J. Timonen, Phys. Rev. E 56, 6042 (1997).

[8] C. S. O'Hern, L. E. Silbert, A. J. Liu, and S. R. Nagel, Phys. Rev. E 68, 011306 (2003).

[9] J.-N. Roux, e-print cond-mat/0405358.

[10] C.-h. Liu and S. R. Nagel, Phys. Rev. Lett. 68, 2301 (1992).

[11] C.-h. Liu and S. R. Nagel, Phys. Rev. B 48, 15646 (1993).

[12] C.-h. Liu, Phys. Rev. B 50, 782 (1994).

[13] M. Leibig, Phys. Rev. E 49, 1647 (1994).

[14] S. Melin, Phys. Rev. E 49, 2353 (1994).

[15] X. Jia, C. Caroli, and B. Velicky, Phys. Rev. Lett. 82, 1863 (1999).

[16] X. Jia and P. Mills, in Powders and Grains 2001, edited by Y. Kishino (Swets and Zeitlinger, Lisse, 2001), pp. 105-112.

[17] B. Gilles and C. Coste, Phys. Rev. Lett. 90, 174302 (2003).

[18] F. Tatsuoka, in Geotechnics for Roads, Rail Tracks and Earth Structures, edited by Correia and Brandle (Balkema, Lisse, 2001), pp. 69-140.

[19] P.-Y. Hicher, J. Geotech. Eng. 122, 641 (1996).

[20] H. Geoffroy, H. di Benedetto, A. Duttine, and C. Sauzéat, in Deformation Characteristics of Geomaterials, edited by H. di Benedetto, T. Doanh, H. Geoffroy, and C. Sauzéat (Swets and Zeitlinger, Lisse, 2003), pp. 353-363.

[21] Y.-C. Chen, I. Ishibashi, and J. T. Jenkins, Geotechnique 38, 23 (1988).

[22] Y.-C. Chen, I. Ishibashi, and J. T. Jenkins, Geotechnique 38, 33 (1988).

[23] T. G. Thomann and R. D. Hryciw, Geotech. Test. J. 13, 97 (1990).

[24] X. Zeng and B. Ni, J. Geotech. Geoenviron. Eng. 125, 741
(1999).

[25] M. Sharifipour, C. Dano, and P.-Y. Hicher, in Proceedings of the Engineering Mechanics 2004 Symposium of the American Society of Civil Engineering, (2004) CD-ROM.

[26] V. Jovicić and M. R. Coop, Geotech. Test. J. 21, 3 (1998).

[27] M. L. Lings and P. D. Greening, Geotechnique 51, 713 (2001).

[28] G. Viggiani and J. H. Atkinson, Geotechnique 45, 149 (1995).

[29] V. Jovicić, M. R. Coop, and M. Simić, Geotechnique 46, 357 (1996).

[30] H. A. Makse, D. L. Johnson, and L. M. Schwartz, Phys. Rev. Lett. 84, 4160 (2000).

[31] C. Moukarzel, Phys. Rev. Lett. 81, 1634 (1998).

[32] A. Tkachenko and T. Witten, Phys. Rev. E 60, 687 (1999).

[33] A. Kasahara and H. Nakanishi, e-print cond-mat/0405169.

[34] J.-N. Roux, Phys. Rev. E 61, 6802 (2000).

[35] L. E. Silbert, D. Ertas, G. S. Grest, T. C. Halsey, and D. Levine, Phys. Rev. E 65, 031304 (2002).

[36] F. Radjai, D. E. Wolf, M. Jean, and J.-J. Moreau, Phys. Rev. Lett. 80, 61 (1998).

[37] C. Goldenberg and I. Goldhirsch, Eur. Phys. J. E 9, 245 (2002).

[38] C. Goldenberg and I. Goldhirsch, Phys. Rev. Lett. 89, 084302 (2002).

[39] J.-N. Roux and G. Combe, C. R. Phys. 3, 131 (2002).

[40] A. Tanguy, J. P. Wittmer, F. Leonforte, and J.-L. Barrat, Phys. Rev. B 66, 174205 (2002).

[41] J.-N. Roux, in Powders and Grains 97, edited by R. P. Behringer and J. T. Jenkins (Balkema, Rotterdam, 1997), pp. 215-218.

[42] E. Somfai, M. van Hecke, and W. van Saarloos (unpublished).

[43] V. F. Nesterenko, J. Appl. Mech. Tech. Phys. 5, 733 (1984).

[44] E. J. Hinch and S. Saint-Jean, Proc. R. Soc. London, Ser. A 455, 3201 (1999).

[45] A. Chatterjee, Phys. Rev. E 59, 5912 (1999).

[46] E. Hascoet and E. J. Hinch, Phys. Rev. E 66, 011307 (2002).

[47] A. Rosas and K. Lindenberg, Phys. Rev. E 68, 041304 (2003).

[48] A. Rosas and K. Lindenberg, Phys. Rev. E 69, 037601 (2004).

[49] J. Duffy and R. D. Mindlin, J. Appl. Mech. 24, 585 (1957).

[50] S. N. Domenico, Geophysics 42, 1339 (1977).

[51] K. L. Johnson, Contact Mechanics (Cambridge University Press, Cambridge, U.K. 1985). 